\title{
Integrated analyses of the transcriptome and small RNA of the hemiparasitic plant Monochasma savatieri before and after establishment of parasite-host association
}

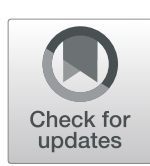

\author{
Lanlan Chen, Qiaosheng Guo, Zaibiao Zhu* (D), Hefang Wan, Yuhao Qin and Hui Zhang
}

\begin{abstract}
Background: Monochasma savatieri is a medicinal root hemiparasitic herb that extracts water and nutrients from the host plant via a haustorium. M. savatieri exhibits an enhanced growth after the establishment of parasite-host associations, but little is known about the molecular mechanism responsible. In this study, endogenous hormones, RNA sequencing and small RNA sequencing analysis were performed on M. savatieri before and after establishment of parasite-host associations.

Results: When grown with the host, decreased contents of jasmonic acid (JA) and indole-3-acetic acid (IAA) and increased abscisic acid (ABA) content were observed in M. savatieri with the established parasitic relationship. When grown with the host, 46,424 differentially expressed genes (DEGs) and 162 differentially expressed miRNAs (DEmiRs) were identified in the comparison between M. savatieri with the established parasitic relationship and without the established parasitic relationship. Analysis of Gene Ontology (GO) and Kyoto Encyclopedia of Genes and Genomes (KEGG) showed that these DEGs and targets of DEmiRs mostly participated in plant hormone signal transduction, starch and sucrose metabolism, carbohydrate metabolism, cell growth and death, and transport and catabolism. Furthermore, correlation analysis of mRNA and miRNA revealed that 10 miRNA-target pairs from novel_mir65, novel_mir40, novel_mir80, miR397-5p_1, novel_mir36, novel_mir25 and novel_mir17 may have important roles in regulating the parasitic development of $M$. savatieri.
\end{abstract}

Conclusions: Our study not only expands the understanding of enhanced growth in M. savatieri after the establishment of parasite-host associations, but also first provides abundant resources for future molecular and genetic studies in M. savatieri.

Keywords: High-throughput sequencing, Parasite-host association, Plant endogenous hormone, Root hemiparasite

\section{Background}

Monochasma savatieri Franch. ex Maxim of Orobanchaceae is a perennial root hemiparasitic herb, which grows well in sunny and hilly terrains of Southeast China and Kyushu (Amakusa Islands), Japan [1-3]. After drying, its

* Correspondence: zhuzaibiao@njau.edu.cn Institute of Chinese Medicinal Materials, Nanjing Agricultural University, Nanjing 210095, China

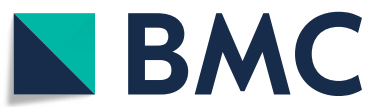

whole plant is necessary for Yanning syrup (one proprietary Chinese medicine), which is commonly applied to cure urinary tract infections, upper respiratory tract infections, and tonsillitis [4]. Currently, wild M. savatieri resources are decreasing sharply, mainly due to the unsustainable use of the wild resources [3]. So far, using tissue culture technique to micropropagate $M$. savatieri has been reported in a few studies $[5,6]$, and the lack of

(c) The Author(s). 2021 Open Access This article is licensed under a Creative Commons Attribution 4.0 International License, which permits use, sharing, adaptation, distribution and reproduction in any medium or format, as long as you give appropriate credit to the original author(s) and the source, provide a link to the Creative Commons licence, and indicate if changes were made. The images or other third party material in this article are included in the article's Creative Commons licence, unless indicated otherwise in a credit line to the material. If material is not included in the article's Creative Commons licence and your intended use is not permitted by statutory regulation or exceeds the permitted use, you will need to obtain permission directly from the copyright holder. To view a copy of this licence, visit http://creativecommons.org/licenses/by/4.0/ The Creative Commons Public Domain Dedication waiver (http://creativecommons.org/publicdomain/zero/1.0/) applies to the data made available in this article, unless otherwise stated in a credit line to the data. 
studies of parasite-host associations limits the establishment of its successful artificial cultivation system. In previous investigations, we studied the growth and anatomical characteristics of $M$. savatieri seedlings before and after establishing parasitic relationships with the host [7]. However, the research on the molecular mechanisms and regulatory networks of $M$. savatieri plants before and after establishing parasitic relationships with the host has not been carried out. This lack of knowledge limits the study for improving the successful parasitization of $M$. savatieri by using reliable technologies. Therefore, exploring the molecular mechanisms is needed to promote further study of the establishment of parasite-host associations to meet the increasing market demand.

As a special organ of parasitic plants, the haustorium is considered as the essence of parasitism [8]. In addition to haustorium-inducing factors, plant hormones also play roles in haustorium formation. In the process of haustorium formation in Cassytha filiformis (Lauraceae), the contents of cytokinins indolepropionic acid (IPA) and zeatin riboside (ZR) were 20 times and 4 times higher than the contents in the control part, respectively, after the parasitic plant attached to the host stem [9]. A study on Cuscuta japonica showed that the synthesized cytokinins were closely related to the development of the haustorium [10]. In addition, a large amount of auxin accumulated in the haustorium forming area of Cuscuta and was excreted from epidermal secretory cells [11].

As an economical and feasible sequencing technology, transcriptome sequencing (RNA-seq) has been widely used to expand sequence databases, and understand differentially expressed genes (DEGs) at the transcriptional and genomic levels. In recent years, RNA-seq has shown great potential in understanding the characteristics of parasitic plants. The dramatic expression patterns of host-specific genes were revealed by analyzing the data generated from RNA-seq of tissues at the interface between parasitic Triphysaria versicolor and its host Medicago truncatula or Zea mays [12]. In addition, comparative transcriptome analysis of three parasitic plants in Orobanchaceae indicated the conservation of chlorophyll synthesis in hemiparasites [13]. Ranjan et al. [14] sequenced the transcriptome of the parasitic weed Cuscuta pentagona and found that parasite genes encoding transporters and enzymes were upregulated during the parasitic process, whereas genes associated with phytohormones were differentially expressed. RNA-seq was also used in a study on haustorium development of the root hemiparasite Santalum album, which suggested that auxin signaling was essential for haustorial initiation [15]. Kim et al. [16] used transcriptome sequencing to characterize mRNA transfer between C. pentagona and Arabidopsis or tomato hosts in a bidirectional manner.

MicroRNA (miRNA) composing of 21-24 nucleotides is a small non-coding RNA that primarily plays a role in translational repression or destabilization [17] by completely or partially binding the target mRNA [18]. In plants, miRNAs represent a group of the best studied small RNAs (sRNAs), and their various roles in development, including leaf growth, flowering and root elongation, have been reported [19, 20]. Recently, miRNAs have attracted attention as cross-species regulators [21, 22]. For example, functional studies in vitro and in vivo demonstrated that the exogenous plant miR168a in rice could regulate the expression of the mammalian gene LDLRAP1 [21]. A study in the parasite Cuscuta campestris-Arabidopsis thaliana pair identified 27 miRNAs of $22 \mathrm{nt}$ in length, which targeted several A. thaliana mRNAs, that were up-regulated in the interface by taking advantage of sRNA sequencing technology [22].

In the present study, we took advantage of RNA-seq and sRNA sequencing technologies to profile the mRNAs and miRNAs in $M$. savatieri roots before and after establishing parasitic relationships to understand the mechanisms by which parasitizing the host promoted $M$. savatieri development. Therefore, the regulatory network of the interactions between DEmiRs and DEGs was constructed by analyzing the DEGs and differentially expressed miRNAs (DEmiRs) obtained from pairwise comparisons. The results of this study provide useful resources to explore the development of hemiparasites and further extend our understanding of miRNAmRNA networks in plants.

\section{Results}

\section{Endogenous hormone content}

To explore the mechanisms underlying the significant growth and development variations in $M$. savatieri before and after parasitizing G. jasminoides (Fig. 1a), we analyzed the endogenous hormone content of $M$. savatieri seedlings. When grown with or without a host, the content of indole-3-acetic acid (IAA), methyl indole-3acetate (ME-IAA) and indole-3-carboxaldehyde (ICA) showed a consistently decreasing trend in the two developmental stages, and the content of ICA was higher than that of IAA and ME-IAA (Fig. $1 \mathrm{~b}$ and c). At 8 WAS, the levels of trans-zeatin (tZ) and cis-zeatin (cZ) in M. savatieri seedlings grown without the host were the highest and were significantly different from the levels in any other samples (Fig. 1d). In the presence of the host, jasmonic acid (JA) content in M. savatieri parasitizing G. jasminoides showed a decrease of $66.20 \%$ compared with the JA content in the M. savatieri before establishing parasite-host associations. Additionally, there was a 


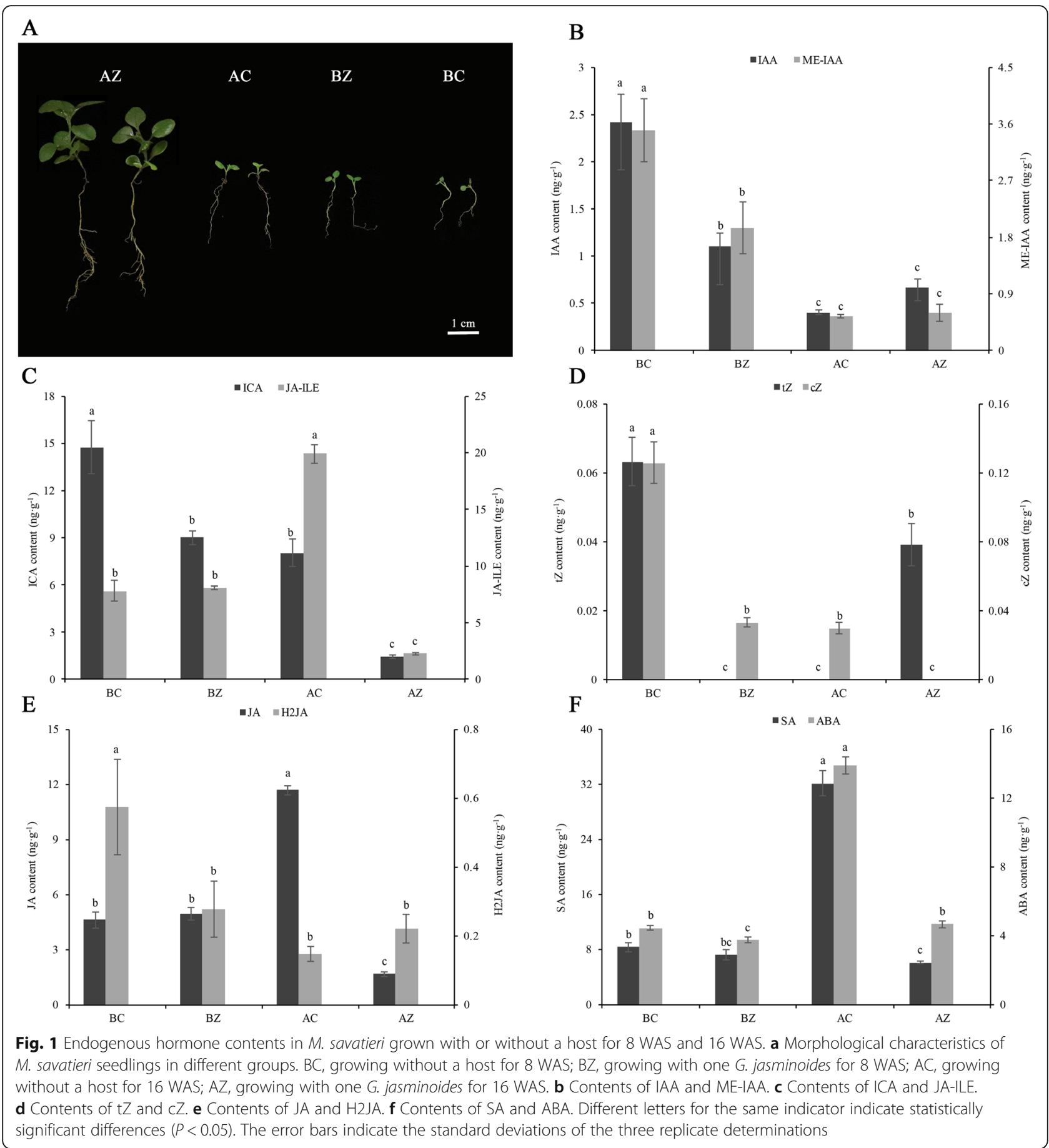

declining trend for dihydrojasmonic acid (H2JA) content after parasite-host associations were established, whereas no significant difference was found (Fig. 1e). At 8 WAS, there was no significant difference in salicylic acid (SA) content in M. savatieri seedlings in the presence or absence of G. jasminoides. At 16 WAS, the SA content of plants growing without $G$. jasminoides was significantly higher than the SA content of post-parasitization plants.
In addition, the establishment of parasite-host associations significantly increased abscisic acid (ABA) content in plants compared with plants growing with $G$. jasminoides for 8 WAS (Fig. 1f).

RNA-seq, sequence assembly and functional annotation A total of twelve library reads of $M$. savatieri were built for RNA-seq to expose the transcriptome differences 
during parasite development using the BGISEQ-500 platform. In this study, $74.27 \mathrm{~Gb}$ of sequence data were obtained from roots of $M$. savatieri plants, which were grown without or with the host for 8 or 16 WAS. After assembly and de redundancy, 167,941 unigenes were obtained with a total length of 249 , 584,355 bp, a GC content of $40.76 \%$, an N50 length of $2474 \mathrm{bp}$, and an average length of $1486 \mathrm{bp}$. The sequence length of these unigenes was between $200 \mathrm{bp}$ and $3000 \mathrm{bp}$, and up to 86,644 unigenes (51.59\%) were larger than $1000 \mathrm{bp}$ (Fig. S1). The ratio of clean reads to total raw reads in all cDNA libraries exceeded $95.85 \%$, and the quality of the sequenced bases is presented in Table 1 . The lowest value of Q20 was $97.62 \%$, and the lowest value of Q30 was $89.72 \%$. The quality of assembled transcripts was evaluated by BUSCO, and the results are shown in Fig. S2. The BUSCO analysis showed that among all the assembled unigenes, of which $22 \%$ were single-copy complete, $76 \%$ were duplicated complete, $1 \%$ were fragmented, and $1 \%$ were missing.

To obtain an overall understanding of the functions of the unigenes that were obtained, all unigenes were searched against the Clusters of Orthologous Groups for Eukaryotic Complete Genomes (KOG), SwissProt, nonredundant protein database (NR), KEGG, nucleotide sequence database (NT) and the Pfam databases. Approximately 116,572 (blast to NR database), 95,387 (blast to NT database), 94,681 (blast to KEGG database), and 91, 301 (blast to Pfam database) unigenes were similar in sequence to known genes (Table 2). By GO analysis, 62, 227 unigenes were annotated and classified into 41 functional groups of three GO categories (Fig. S2). Among the GO terms, catalytic activity and molecular function
Table 2 Statistical summary of functional annotation of $M$. savatieri unigenes

\begin{tabular}{lll}
\hline Annotated databases & Annotated number & Annotated percentage \\
\hline NR & 116,572 & $69.41 \%$ \\
NT & 95,387 & $56.80 \%$ \\
Swissprot & 88,877 & $52.92 \%$ \\
KEGG & 94,681 & $56.38 \%$ \\
KOG & 96,800 & $57.64 \%$ \\
Pfam & 91,301 & $54.36 \%$ \\
GO & 62,227 & $37.05 \%$ \\
Total Annotated & 126,048 & $75.05 \%$ \\
\hline
\end{tabular}

accounted for the largest proportion in functional groups and GO categories, respectively.

\section{Identification of DEGs}

To present the number of DEGs in different comparisons, a Venn diagram was built (Fig. 2a). Among these four comparisons, BZ/AZ had the largest number of DEGs $(46,424)$, followed by AC/AZ (43,746) and $\mathrm{BC} / \mathrm{AC}(38,111)$, and $\mathrm{BC} / \mathrm{BZ}$ had the smallest number of DEGs $(26,804)$. In addition, 4351 DEGs were identified in all four comparisons. There were 11,778 up-regulated genes and 26,333 down-regulated genes detected under AC compared with BC. Comparison of BZ with AZ revealed 27,098 up-regulated genes and 19,326 down-regulated genes (Fig. $2 \mathrm{~b}$ and Additional file 2). To have a visual understanding of the overall expression profile, a heat map was constructed (Fig. 2c). By analyzing gene expression, a large portion of genes with higher levels in $\mathrm{BC}$ were found to exhibit lower levels in AC. Furthermore,

Table 1 Statistical summary of the transcriptome assembly for M. savatieri

\begin{tabular}{llllllll}
\hline Sample & Total raw reads $(M)$ & Total clean reads (M) & Clean reads number & Clean reads ratio(\%) & Q20(\%) & Q30(\%) & Total mapping(\%) \\
\hline BC 1 & 64.44 & 61.88 & $61,877,098$ & 96.03 & 97.71 & 90.11 & 89.39 \\
BC 2 & 64.44 & 61.94 & $61,944,568$ & 96.13 & 97.73 & 90.28 & 89.36 \\
BC 3 & 64.4 & 61.84 & $61,843,080$ & 96.03 & 97.83 & 90.53 & 84.9 \\
BZ 1 & 64.44 & 61.73 & $61,727,048$ & 95.79 & 97.71 & 90.17 & 89.88 \\
BZ 2 & 64.44 & 61.93 & $61,932,718$ & 96.11 & 97.81 & 90.51 & 89.7 \\
BZ 3 & 64.43 & 62.01 & $62,009,278$ & 96.24 & 97.83 & 90.54 & 87.89 \\
AC 1 & 64.4 & 62.03 & $62,029,070$ & 96.31 & 97.83 & 90.47 & 89.16 \\
AC 2 & 64.44 & 61.94 & $61,937,642$ & 96.12 & 97.78 & 90.31 & 88.4 \\
AC 3 & 64.41 & 61.74 & $61,742,462$ & 95.85 & 97.62 & 89.72 & 90.01 \\
AZ 1 & 64.44 & 61.79 & $61,785,376$ & 95.88 & 97.63 & 89.85 & 89.67 \\
AZ 2 & 64.4 & 61.86 & $61,859,516$ & 96.06 & 97.78 & 90.34 & 90.7 \\
AZ 3 & 64.46 & 62.16 & $62,155,874$ & 96.43 & 97.88 & 90.7 & 87.79
\end{tabular}

BC, growing without a host for 8 WAS; BZ, growing with one G. jasminoides for 8 WAS; AC, growing without a host for 16 WAS; AZ, growing with one G. jasminoides for 16 WAS 
A

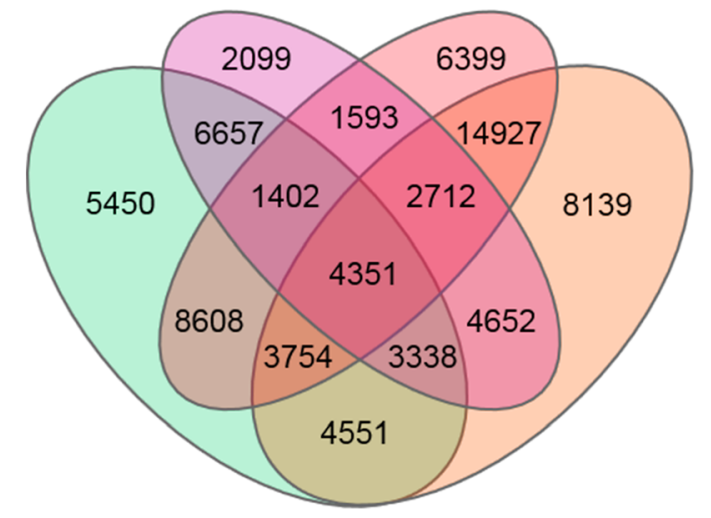

C

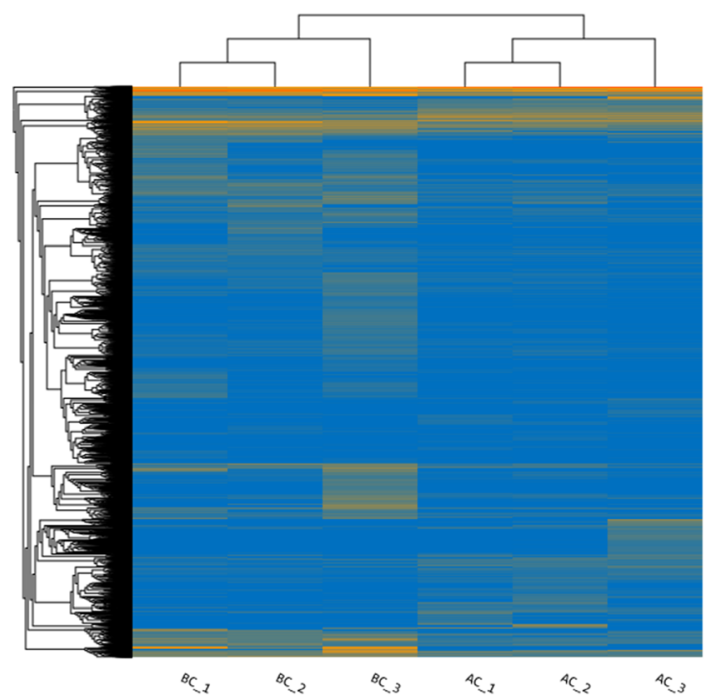

B

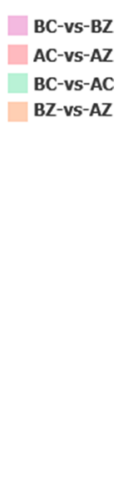

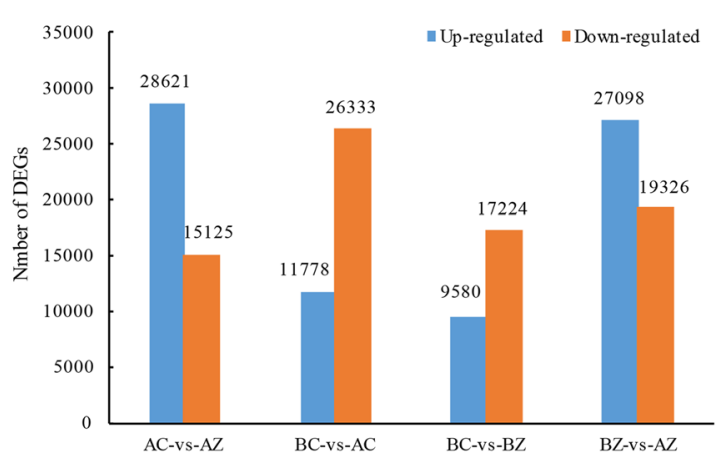

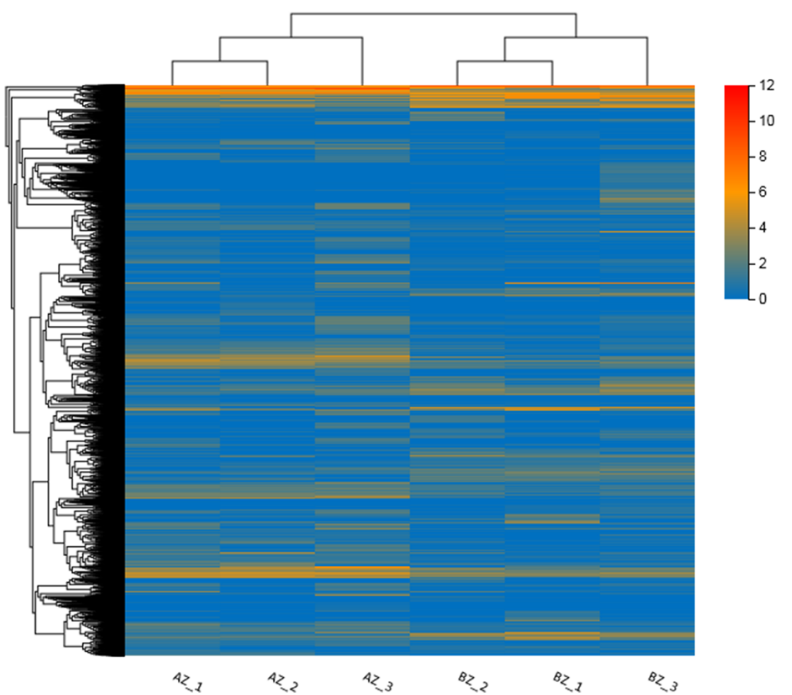

Fig. 2 Gene expression profiles of M. savatieri grown with or without a host for 8 WAS and 16 WAS. a Venn diagram of common and unique DEGs among different comparisons. $\mathbf{b}$ Expression patterns of DEGs among different comparisons. $\mathbf{c}$ Expression profiles and cluster analysis of total DEGs in BC/AC and BZ/AZ comparisons. BC, growing without a host for 8 WAS; BZ, growing with one $G$. jasminoides for 8 WAS; AC, growing without a host for 16 WAS; AZ, growing with one G. jasminoides for 16 WAS

most of the DEGs presented larger expression differences in $\mathrm{BZ}$ and $\mathrm{AZ}$ than in $\mathrm{BC}$ and $\mathrm{AC}$.

\section{Functional classification and enrichment analysis of the DEGs}

To further understand the DEGs, we used GO and KEGG pathway analysis to evaluate the DEGs (Fig. 3). By analyzing these three comparisons, the annotated DEGs were observed to be enriched for several specific $\mathrm{GO}$ terms. The number of DEGs in the $\mathrm{BZ}$ vs AZ comparison was greater than the number of DEGs in the $B C$ vs $A C$ and $A C$ vs $A Z$ comparisons. With regard to each comparison, most of the DEGs were enriched in the cellular component category, with the highly represented GO terms classified into "cell", "membrane", "cell part", "organelle" and "membrane part". The GO terms were significantly classified into "metabolic process" and "cellular process" in the biological process category and "catalytic activity" and "binding" in the molecular function category (Fig. 3a-c and Additional file 3).

To gain more insights into the metabolic pathways and biological functions of the DEGs, an enrichment analysis was performed on the basis of the KEGG database. There were 14,375 DEGs detected in the BC vs AC comparison, $15,320 \mathrm{DEGs}$ in the $\mathrm{BZ}$ vs $\mathrm{AZ}$ comparison, and 14,659 DEGs in the AC vs AZ comparison. In these comparisons, the top 20 pathways that were enriched by the most significant differentially expressed genes are given (Fig. 3d-f). In the $\mathrm{BZ}$ vs $\mathrm{AZ}$ and $\mathrm{AC}$ vs $\mathrm{AZ}$ comparisons, the DEGs were mostly enriched in "plant hormone signal transduction", "starch and sucrose metabolism", "phenylpropanoid biosynthesis", "MAPK signaling pathway-plant", "galactose metabolism", "zeatin biosynthesis", and "plant-pathogen interaction". Notably, 


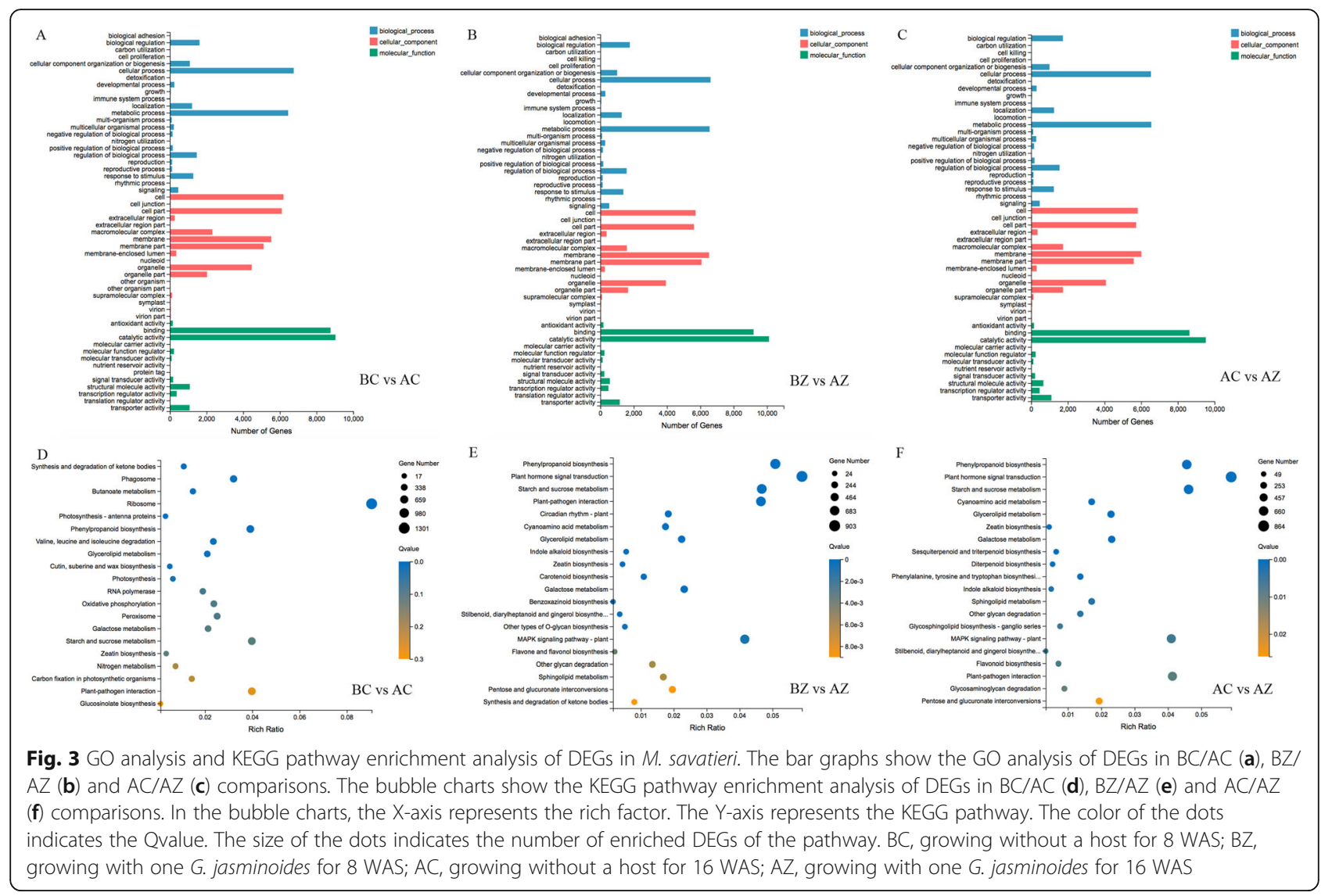

"circadian rhythm-plant" and "carotenoid biosynthesis" were only enriched in the BZ vs AZ comparison. In the $\mathrm{BC}$ vs $\mathrm{AC}$ comparison, the DEGs were significantly enriched in "photosynthesis-antenna proteins", "phagosome", "butanoate metabolism", "ribosome", and "synthesis and degradation of ketone bodies". Moreover, "nitrogen metabolism", "carbon fixation in photosynthetic organisms", "photosynthesis", and "cutin, suberine and wax biosynthesis" were significantly enriched only in the $\mathrm{BC}$ vs $\mathrm{AC}$ comparison.

\section{Transcription factors related to the biological processes before and after establishing parasitic relationship with the host}

Since $M$. savatieri established a parasitic relationship with the host during growth with G. jasminoides, the DEGs related to the biological processes in the $\mathrm{BZ}$ vs AZ comparison were then selected for further analysis. Here, 66 transcription factors (TFs), which might have the putative regulatory role in the establishment of parasite-host associations, were identified among these DEGs. The TF families included WRKY, TCP, PBF-2like, NAC, MYB, MADS, GRAS, G2-like, FAR1, C3H, C2H2, C2C2-GATA, C2C2-Dof, BES1, ARF, AP2EREBP, and ABI3VP1 (Table S1). Among these TFs, the
MYB family contained the largest number of DEGs, of which 7 putative MYBs were up-regulated under BZ/AZ. The WRKY family had 8 DEGs, which followed the MYB family. Moreover, the expression levels of all 8 putative WRKYs decreased under AZ compared to BZ, suggesting that the TFs may negatively regulate the establishment of parasite-host associations.

\section{DEG network and pathways related to establishment of the parasitic relationship}

To understand the underlying mechanism of $M$. savatieri-enhanced parasitic development, the DEGs only specifically expressed in the BZ vs AZ comparison and significantly enriched in "plant-pathogen interaction", "carotenoid biosynthesis", "phenylpropanoid biosynthesis", "plant hormone signal transduction", and "endocytosis" were compared with the STRING database by DIAMOND, and the interaction between genes was obtained by homology with known proteins. In total, 46 interactions among 50 genes were identified from 500 DEGs in the rebuilt gene network (Fig. 4). Among the 50 identified genes, three DEGs, namely, MsPTI1, $M s H A L 3 A$, and MsCURL3, interacted with other DEGs more frequently, indicating that they may have key roles in the establishment of the M. savatieri-host association. 


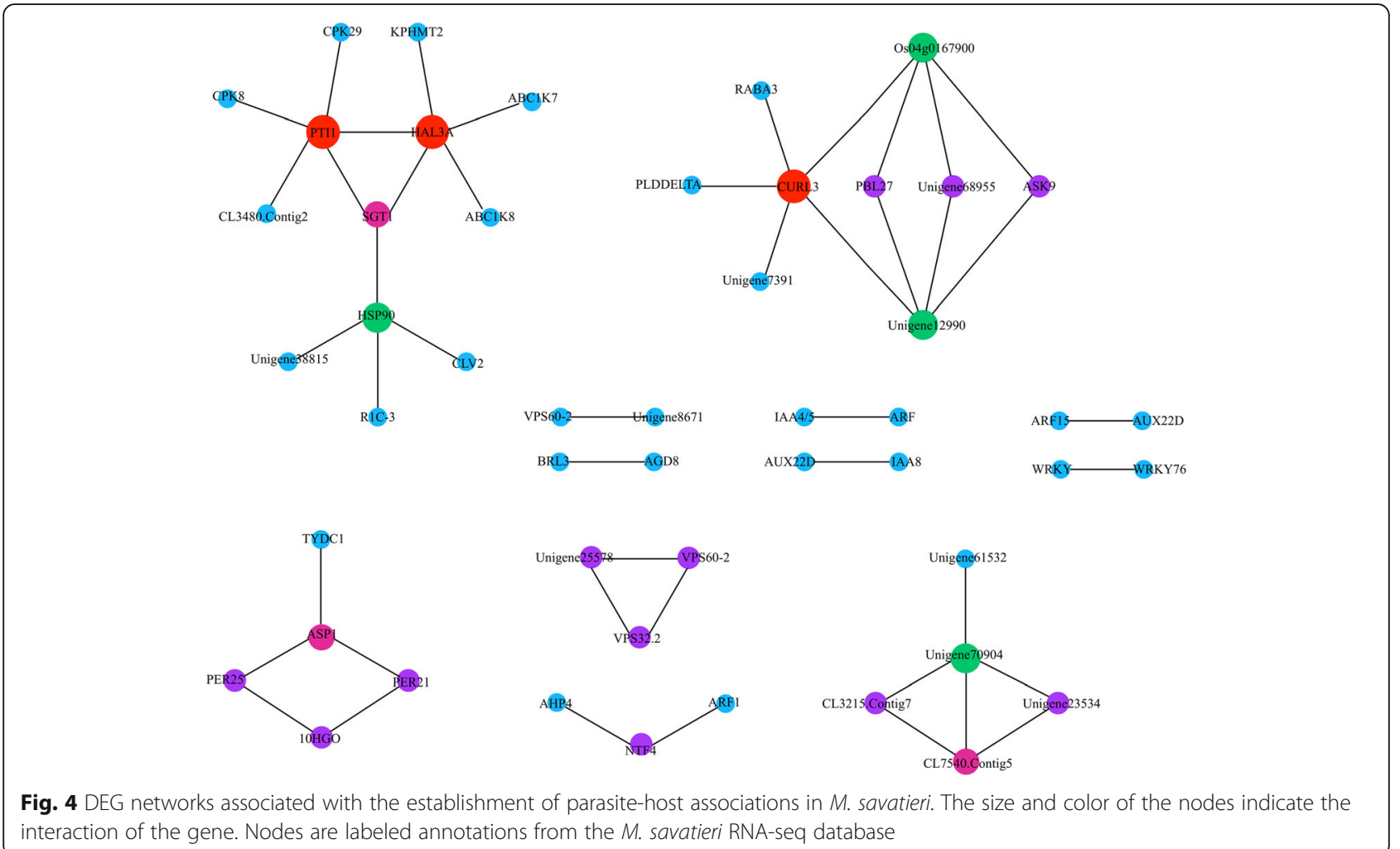

By mapping to the KEGG database, the three DEGs MsPTI1, MsHAL3A, and MsCURL3 were found to be involved in "metabolic pathways" and "plant hormone signal transduction". M. savatieri exhibited enhanced growth after establishing parasitic relationships with the host, and the above results indicated that plant hormones played important roles in the establishment of $M$. savatieri-host associations. Thus, the DEGs only specifically expressed in the BZ vs AZ comparison were listed in the "plant hormone signal transduction" and "citrate cycle" pathways to obtain detailed information (Fig. 5). In the citrate cycle, 8 DEGs (4 up-regulated and 4 downregulated) were involved in the processes of the cycle between citrate, cis-acotinate and isocitrate and

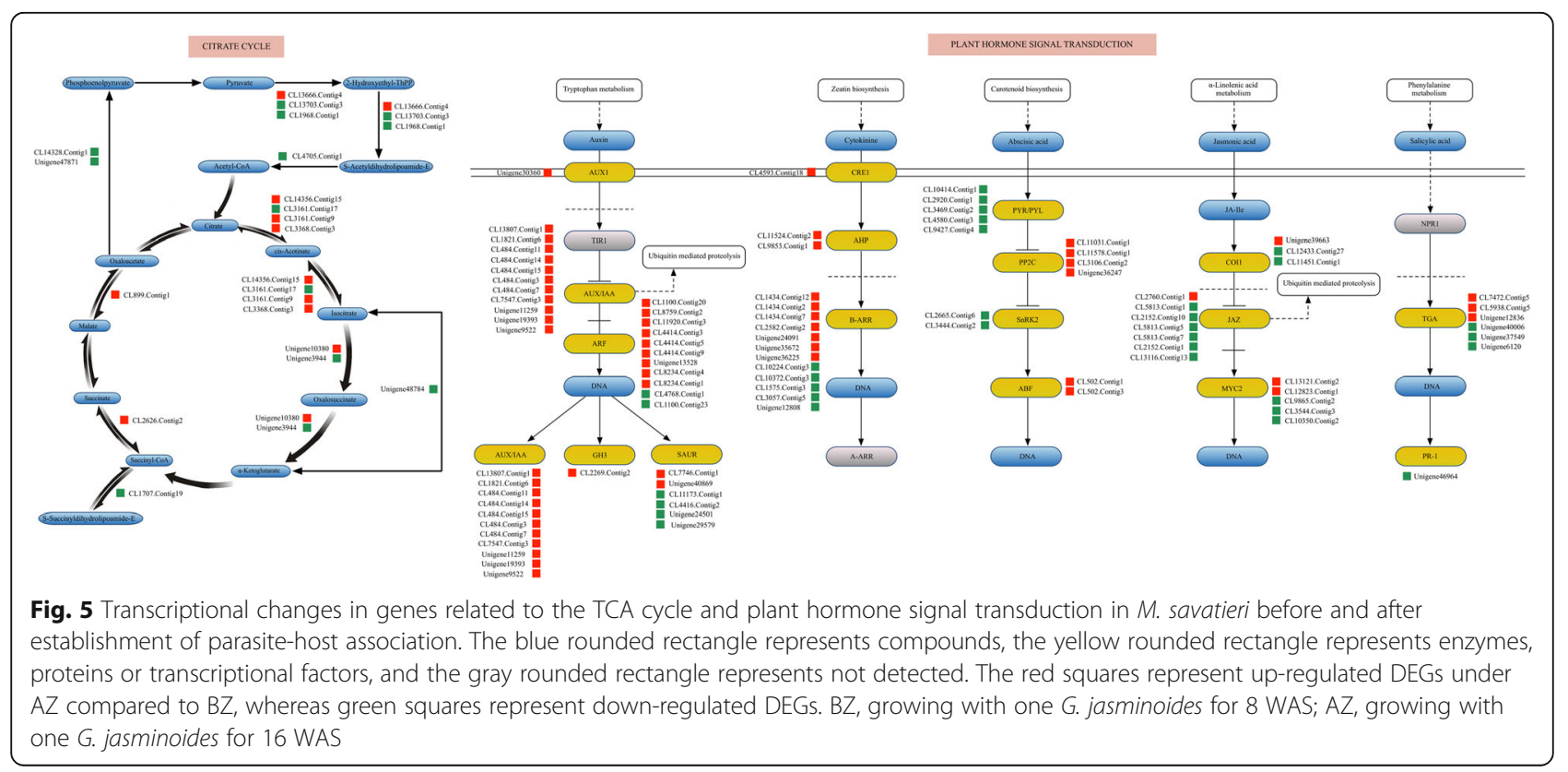


biosynthesis of acetyl-CoA. In plant hormone signal transduction, the number of genes involved in auxin signal transduction was the highest, and the expression levels of these genes were up-regulated after establishing parasitic relationships with the host. In addition, $2 M S C R E 1$ putative target genes (CL11524. Contig2 and CL9853. Contig1) were identified in cytokinine signal transduction.

\section{qRT-PCR validation of DEGs associated with parasite development}

Establishing parasitic relationships with the host was achieved by a series of processes, and numerous genes were involved in these steps. In the experiment, we performed validation of selected 14 DEGs by quantitative real-time PCR (qRT-PCR). The selected DEGs were predicted to play a role in starch and sucrose metabolism (Unigene11723, Unigene28565, Unigene25653, Unigene35991, Unigene32595), hormone signal transduction (Unigene9451, Unigene21852, Unigene29438, Unigene60184), and cell part (Unigene36901, Unigene470, Unigene5067, Unigene10023, Unigene25917). The qRTPCR analysis showed that the expression patterns of these genes were consistent with the expression pattern of the corresponding transcriptome data (Fig. S3).

Among the genes related to starch and sucrose metabolism (Fig. S3a and b), the expression levels of Unigene11723 and Unigene28565 increased under AZ compared with BZ and AC. The expression of Unigene25653, Unigene35991 and Unigene32595 decreased under $\mathrm{AZ}$ relative to $\mathrm{BZ}$ and $\mathrm{AC}$. The expression levels of four genes involved in hormone signal transduction were significantly different before and after establishing parasitic relationships with the host (Fig. S3c and d). In addition, qRT-PCR analysis showed that Unigene9451,
Unigene21852 and Unigene29438 exhibited much higher expression levels in AZ than the other three groups, while regarding Unigene60184, its expression level decreased at least 20 -fold. In the cell part, four genes (Unigene36901, Unigene470, Unigene5067 and Unigene10023) exhibited a higher expression level in AZ than AC, while Unigene 25917 exhibited a significantly lower expression level in AZ than BZ (Fig. S3e and f).

\section{Small RNA sequencing and category annotation}

A total of twelve sRNA libraries (BC, BZ, AC, and AZ, each repeated three times) of $M$. savatieri were prepared from the roots of $M$. savatieri to reveal the miRNA differences in parasite development. In the present study, 21, 527,722 to $30,095,656$ raw tags were generated from each library, and 20,667,261 to 28,872,904 clean tags per library were obtained after removing low-quality tags (Table 3 ). The lowest value of Q20 was $98.6 \%$, and the percentage of mapped tags in all sRNA libraries exceeded $41.1 \%$. The sequence length of these small RNAs ranged from $15 \mathrm{nt}$ to $30 \mathrm{nt}$, of which the number of small RNAs with a length of $24 \mathrm{nt}$ was the largest (Fig. S4). According to the Rfam and GenBank databases, we annotated the sequences from all $M$. savatieri libraries and described the counts of different categories (Table 4). Among these libraries, sequences matching to mature sRNA were the most abundant class, which accounted for almost $2-11 \%$ after excluding the unmapping sequences.

\section{Discovery of known and novel miRNAs}

There were 110 known miRNAs identified from small RNA sequences of $M$. savatieri after comparing the sequences with the known mature miRNAs in the miRBase. With the use of miRA, 128 sequences were

Table 3 Statistical summary of small RNA sequencing data for M. savatieri

\begin{tabular}{llllllll}
\hline Sample & $\begin{array}{l}\text { Raw tag } \\
\text { count }\end{array}$ & $\begin{array}{l}\text { Short valid length } \\
\text { tag }\end{array}$ & $\begin{array}{l}\text { Clean tag } \\
\text { count }\end{array}$ & $\begin{array}{l}\text { Q20 of clean tag } \\
\text { (\%) }\end{array}$ & $\begin{array}{l}\text { Percentage of clean } \\
\text { tag (\%) }\end{array}$ & $\begin{array}{l}\text { Mapped } \\
\text { tag }\end{array}$ & $\begin{array}{l}\text { Mapped tag } \\
\text { percentage (\%) }\end{array}$ \\
\hline BC 1 & $28,103,827$ & $1,137,232$ & $26,100,927$ & 98.6 & 92.87 & $14,883,410$ & 57.02 \\
BC 2 & $28,343,426$ & $1,214,022$ & $26,039,115$ & 99.1 & 91.87 & $15,582,358$ & 59.84 \\
BC 3 & $29,717,549$ & 372,975 & $26,924,615$ & 99.1 & 90.6 & $12,956,213$ & 48.12 \\
BZ 1 & $30,095,656$ & 471,619 & $28,587,339$ & 99.1 & 94.99 & $16,375,175$ & 57.28 \\
BZ 2 & $28,294,198$ & $1,028,007$ & $26,246,425$ & 99.2 & 92.76 & $17,828,594$ & 67.93 \\
BZ 3 & $21,527,722$ & 71,884 & $20,667,261$ & 99.0 & 96 & $10,207,888$ & 49.39 \\
AC 1 & $28,378,126$ & 180,436 & $26,906,040$ & 99.1 & 94.81 & $12,476,677$ & 46.37 \\
AC 2 & $28,251,907$ & 119,769 & $27,298,942$ & 99.1 & 96.63 & $12,509,082$ & 45.82 \\
AC 3 & $29,622,162$ & 109,009 & $28,559,486$ & 99.0 & 96.41 & $14,494,379$ & 50.75 \\
AZ 1 & $29,288,996$ & 85,248 & $28,368,014$ & 98.9 & 96.86 & $11,660,443$ & 41.10 \\
AZ 2 & $29,997,041$ & 113,593 & $28,872,904$ & 99.1 & 96.25 & $13,248,923$ & 45.89 \\
AZ 3 & $29,759,347$ & 104,677 & $28,539,455$ & 99.0 & 95.9 & $11,913,184$ & 41.74
\end{tabular}

BC, growing without a host for 8 WAS; BZ, growing with one G. jasminoides for 8 WAS; AC, growing without a host for 16 WAS; AZ, growing with one G. jasminoides for 16 WAS 
Table 4 Summary of general annotation of clean sequences for small RNAs in M. savatieri

\begin{tabular}{lllllllllll}
\hline Type & & mature & snRNA & rRNA & snoRNA & tRNA & intron & intergenic & precursor & unmap \\
\hline BC-1 & Count & $\mathbf{1 , 8 1 9 , 3 1 6}$ & $\mathbf{8 4 9 9}$ & $\mathbf{1 5 4 , 0 0 7}$ & $\mathbf{2 7 1 2}$ & $\mathbf{1 4 5 9}$ & $\mathbf{1 8 7}$ & $\mathbf{8 9 , 3 5 1}$ & $\mathbf{1 7 , 4 8 7}$ & $\mathbf{1 1 , 1 8 1 , 8 5 1}$ \\
\hline BC-2 & Count & $1,885,269$ & 5196 & 167,570 & 2252 & 1270 & 177 & 198,832 & 20,579 & $10,417,322$ \\
BC-3 & Count & 710,966 & 12,272 & 316,971 & 4057 & 5614 & 389 & 166,410 & 8084 & $13,915,066$ \\
BZ-1 & Count & $3,227,181$ & 10,792 & 132,903 & 2024 & 1379 & 672 & 231,326 & 15,850 & $12,178,998$ \\
BZ-2 & Count & $1,529,809$ & 6917 & 244,911 & 3695 & 1386 & 117 & 117,732 & 19,097 & $8,381,136$ \\
BZ-3 & Count & 511,765 & 10,647 & 363,318 & 6421 & 7966 & 152 & 165,638 & 7130 & $10,418,194$ \\
AC-1 & Count & 489,518 & 13,630 & 274,587 & 3992 & 9445 & 1321 & 208,337 & 6475 & $14,381,159$ \\
AC-2 & Count & $1,151,818$ & 11,542 & 130,686 & 2078 & 3102 & 1049 & 288,352 & 9469 & $14,760,687$ \\
AC-3 & Count & $1,109,687$ & 9683 & 205,523 & 2015 & 2559 & 297 & 216,271 & 21,169 & $14,026,941$ \\
AZ-1 & Count & 637,039 & 22,516 & 309,291 & 4168 & 12,149 & 243 & 194,864 & 9588 & $16,651,139$ \\
AZ-2 & Count & 823,625 & 19,333 & 351,638 & 3178 & 6251 & 128 & 292,921 & 13,832 & $15,578,629$ \\
AZ-3 & Count & 855,925 & 18,067 & 220,546 & 2907 & 4628 & 177 & 286,809 & 10,854 & $16,588,191$ \\
\hline
\end{tabular}

$\mathrm{BC}$, growing without a host for 8 WAS; BZ, growing with one G. jasminoides for 8 WAS; AC, growing without a host for 16 WAS; AZ, growing with one G. jasminoides for 16 WAS

identified as candidates for novel miRNAs. As shown in Fig. S5, the first base distribution of known and novel miRNAs in $M$. savatieri was analyzed. In the identified miRNAs, the percent of the first base was not evenly distributed. The $U$ percent in the first base of most known and novel miRNAs was more than $25 \%$. Venn diagrams were constructed to represent the numbers of overlapping and specific miRNAs from each group (Fig. 6a and b). Among the four libraries, the number of known and novel miRNAs identified in each library was 69 and 119, respectively. Family analysis revealed that of the known miRNAs in $M$. savatieri, 110 miRNAs were from 30 families (Fig. 6c). Among them, the miR166 family had 13 miRNA members and was the largest family of miRNAs, followed by the miR396 family, which had 11 miRNA members. In addition, 14 families contained
A

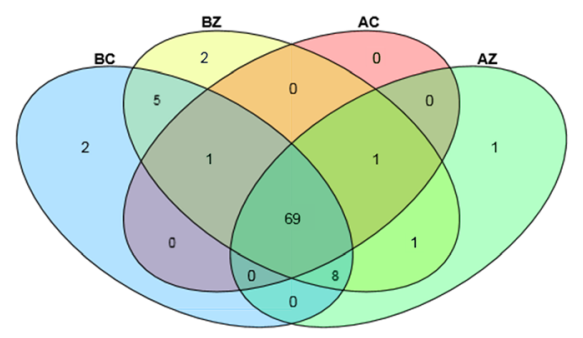

C

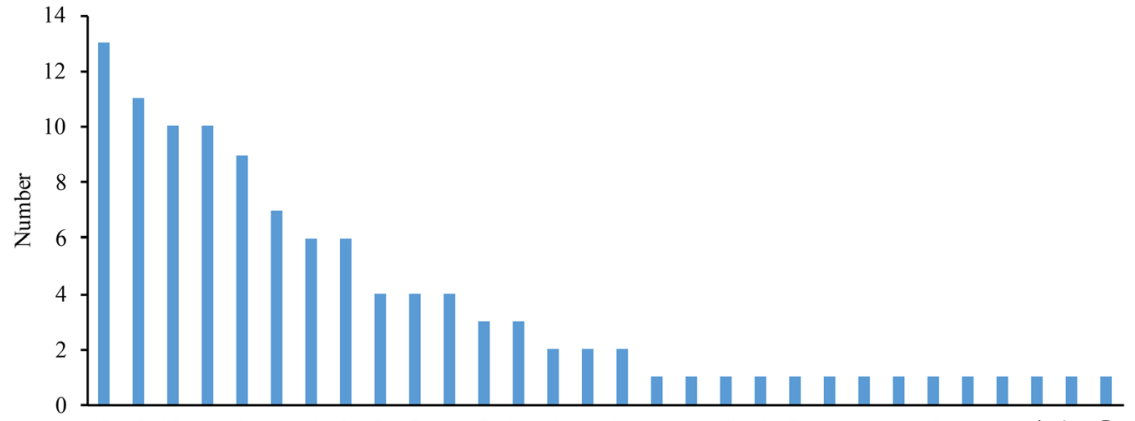

B

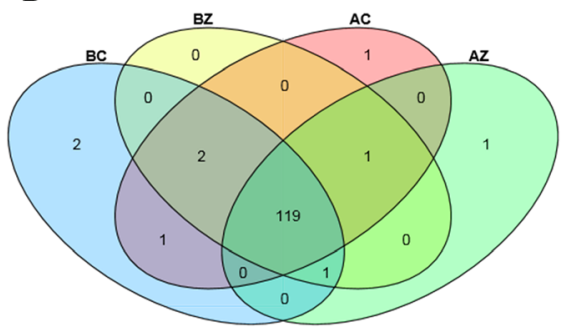

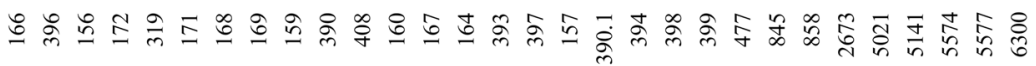

miRNA family

Fig. 6 Identification of miRNAs in M. savatieri. a Venn diagrams of known miRNAs. $\mathbf{b}$ Venn diagrams of novel miRNAs. c Number of known miRNAs in each miRNA family. BC, growing without a host for 8 WAS; BZ, growing with one G. jasminoides for 8 WAS; AC, growing without a host for 16 WAS; AZ, growing with one G. jasminoides for 16 WAS 
only one miRNA member, such as miR157 and miR398 (Fig. 6c).

\section{Screening for DEmiRs}

To determine the differences in miRNA expression in $M$. savatieri before and after establishing parasitic relationship with the host, comparative analyses were performed between $\mathrm{AC}$ and $\mathrm{AZ}, \mathrm{BC}$ and $\mathrm{AC}, \mathrm{BC}$ and $\mathrm{BZ}$, and $\mathrm{BZ}$ and $\mathrm{AZ}$. In general, $198 \mathrm{DEmiRs}$ were detected by using DEGseq. In comparison with $\mathrm{AC}$, the number of down-regulated and up-regulated miRNAs detected in the AZ was 29 and 49, respectively. Comparison of $\mathrm{BZ}$ with $\mathrm{AZ}$ revealed 55 down-regulated miRNAs and 107 up-regulated miRNAs in the AZ (Fig. 7a and Additional file 4). In addition, a general overview of the DEmiRs screened between the four comparison groups was visualized in a heat map (Fig. 7b). According to the expression analysis, most of the DEmiRs under the $\mathrm{BC}$ vs $A C$ comparison were found to have similar expression patterns under the $\mathrm{BZ}$ vs $\mathrm{AZ}$ comparison. In addition, most DEmiRs exhibited larger expression differences in the AC vs $\mathrm{AZ}$ comparison than in the $\mathrm{BC}$ vs $\mathrm{BZ}$ comparison.

\section{Functional annotation of DEmiR target genes}

To further understand the DEmiRs, we used GO and KEGG pathway analysis to evaluate the DEmiR target genes (Fig. 8). By analyzing the three comparisons, the annotated DEmiR target genes were observed to be enriched for several specific GO terms. The number of annotated DEmiR targets in the $\mathrm{BZ}$ vs AZ comparison was greater than the number of annotated DEmiR targets in the $\mathrm{BC}$ vs $\mathrm{AC}$ and $\mathrm{AC}$ vs $\mathrm{AZ}$ comparisons. With regard to each comparison, most DEmiR targets were enriched in the cellular component category, with the highly represented GO terms classified into "cell", "membrane", "cell part", "organelle" and "membrane part". The GO terms were significantly classified into "single-organism process", "metabolic process" and "cellular process" in the biological process category and "catalytic activity" and "binding" in the molecular function category (Fig. 8a-c).

In the KEGG pathway, the number of DEmiR targets identified in the $\mathrm{BC}$ vs $\mathrm{AC}, \mathrm{BZ}$ vs $\mathrm{AZ}$, and $\mathrm{AC}$ vs AZ comparisons were 115,197 , and 85 , respectively (Additional file 5). The top 20 pathways that were enriched by the most significant DEmiR targets in the three comparisons are given (Fig. 8d-f). Among all these comparisons, the DEmiR targets were mostly enriched in "RNA polymerase", "metabolic pathways", "biosynthesis of secondary metabolites", "endocytosis", "oxidative phosphorylation", "photosynthesis", and "pentose and glucuronate interconversions". Notably, "purine metabolism", "propanoate metabolism", "MAPK signaling pathway-plant", "indole alkaloid biosynthesis", "glycolysis/gluconeogenesis" and "biosynthesis of amino acids" were both enriched in the BZ vs AZ and AC vs AZ comparisons. Moreover, "ribosome", "carbon fixation in photosynthetic organisms", "monoterpenoid biosynthesis" and "flavonoid biosynthesis" were significantly enriched only in the $\mathrm{BC}$ vs AC comparison.

qRT-PCR validation of DEmiRs and targets associated with parasite development

Through qRT-PCR, 6 known (miR172a_3, miR156_2, miR396g-3p, miR171b-3p_3, miR397-5p_1, miR396h)
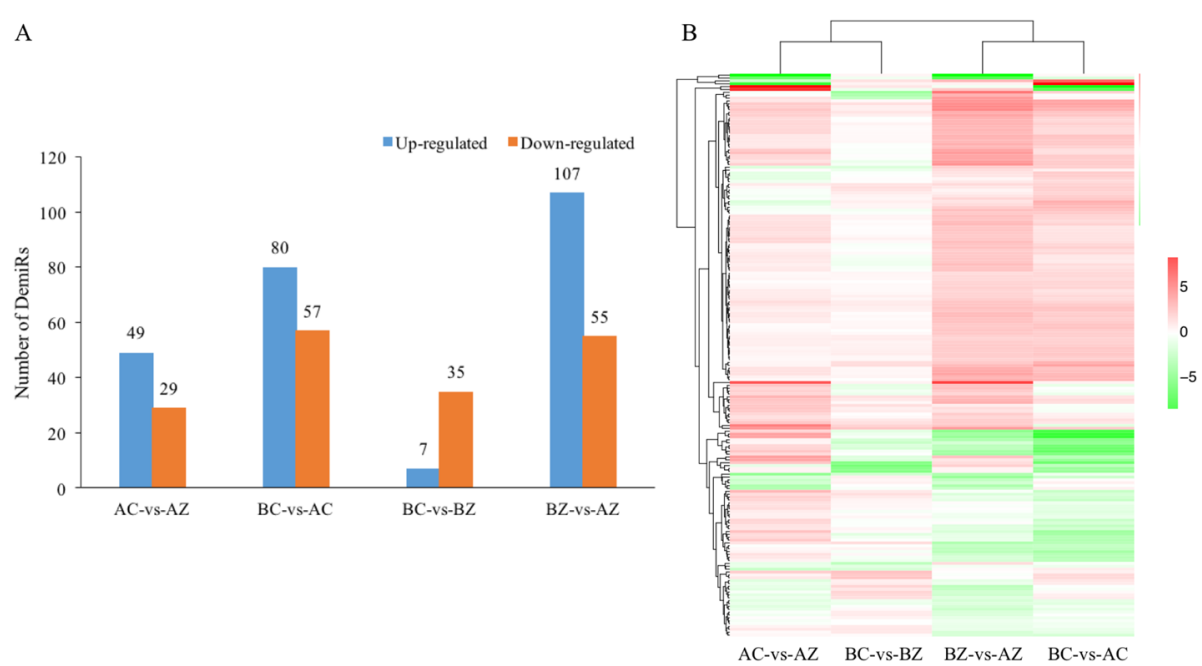

Fig. 7 Changes in miRNA expression profiles of M. savatieri before and after establishment of parasite-host association. a The expression patterns of DEmiRs among different comparisons. $\mathbf{b}$ Cluster analysis of total DEmiRs among different comparisons. BC, growing without a host for 8 WAS; BZ, growing with one G. jasminoides for 8 WAS; AC, growing without a host for 16 WAS; AZ, growing with one G. jasminoides for 16 WAS 


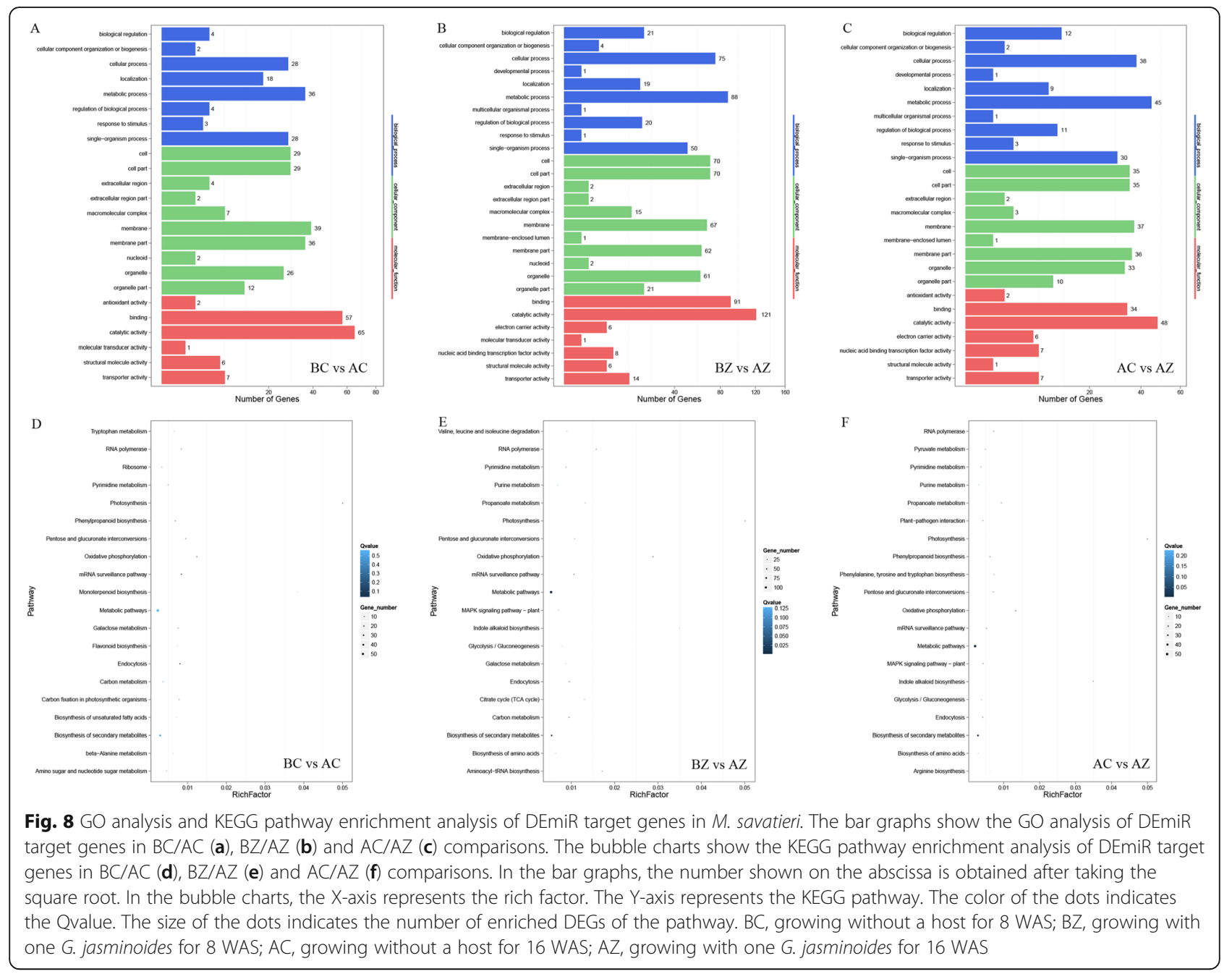

and 9 novel (novel_mir80, novel_mir50, novel_mir46, novel_mir113, novel_mir99, novel_mir49, novel_mir87, novel_mir40, novel_mir65) DEmiRs were selected to analyze the expression levels. These selected miRNA targets were predicted to be associated with biological regulation, membrane and organelle, and metabolism. Among biological regulation-related miRNAs (Fig. S6a and b), the expression levels of novel_mir50 and miR396g-3p increased substantially in M. savatieri seedlings after parasitizing G. jasminoides, while the expression level of novel_mir80 showed a large decrease. With regard to membrane- and organelle-related miRNAs (Fig. S6c and d), the expression of miR171b-3p_3 decreased under $\mathrm{AZ}$ relative to $\mathrm{BZ}$ and $\mathrm{AC}$, while the expression of novel_mir99 showed a significant increase after establishment of parasite-host association. The five metabolism-related miRNAs (novel_mir49, novel_mir87, novel_mir40, novel_mir65, miR396h) exhibited higher expression levels in AZ than the other three groups (Fig. S6e and f).
To further understand the regulation of miRNAs and targets associated with the establishment of parasitic relationships, the expression levels of the selected miRNA targets were analyzed. These chosen target genes were predicted to be associated with biological regulation (CL9918.Contig2, CL14121.Contig1, Unigene14700, CL7316.Contig4, CL219.Contig5), membrane and organelle (Unigene4922, CL1529.Contig2, CL14340.Contig10, Unigene652, Unigene34515), and metabolism (Unigene7334, CL9246.Contig2, Unigene21854, Unigene8689, CL527.Contig67). The 15 target genes showed different relative expression levels in $M$. savatieri seedlings before and after parasitizing the host (Fig. S7). Five target genes (CL9918.Contig2, CL14340.Contig10, Unigene7334, CL9246.Contig2, CL527.Contig67) were strongly negatively correlated with the corresponding miRNAs. The expression level of CL9918.Contig2 was up-regulated after establishment of the parasite-host association, while its corresponding miRNA (miR156_2) was down-regulated. In addition, six target genes 
(Unigene14700, CL14121.Contig1, CL219.Contig5, Unigene34515, Unigene21854, Unigene8689) were strongly positively correlated with the corresponding miRNAs. The expression level of Unigene14700 was downregulated after parasitizing the host, and its corresponding miRNA (novel_mir80) was also down-regulated.

\section{Correlation analysis of co-differentially expressed miRNAs and targets}

In this research, the largest number of co-differentially expressed miRNAs and targets was observed in the BZ vs $\mathrm{AZ}$ comparison, including 58 negative correlations and 260 positive correlations. In the $\mathrm{BC}$ vs $\mathrm{AC}$ comparison, 30 miRNAs and 77 targets were co-differentially expressed (Table 5). To explore the biological functions and distributions of these associated genes between miRNAs and targets in M. savatieri, GO function annotations and KEGG pathway analysis were performed. The number of annotated genes for the same GO terms in the BZ vs AZ comparison was greater than the annotated genes in the $\mathrm{BC}$ vs $\mathrm{AC}$ comparison (Fig. 9a and b). Notably, "negative regulation of biological process" was detected only in the $\mathrm{BC}$ vs $\mathrm{AC}$ comparison, while "positive regulation of biological process" was detected only in the $\mathrm{BZ}$ vs $\mathrm{AZ}$ comparison. Moreover, the associated targets involved in "signaling", "cell junction", "protein binding transcription factor activity", "membraneenclosed lumen", "molecular transducer activity", "extracellular region" and "symplast" were specifically represented in the BZ vs AZ comparison (Fig. 9a and b). With regard to the KEGG pathway, the associated targets in the $\mathrm{BZ}$ vs $\mathrm{AZ}$ and $\mathrm{BC}$ vs $\mathrm{AC}$ comparisons were both significantly enriched in "cell growth and death", "transport and catabolism", "signal transduction", "substance dependence", "carbohydrate metabolism", "development" and "environmental adaptation", but the number of the associated genes for most of the same KEGG terms in the BZ vs AZ comparison was more than the number of the associated genes for most of the same KEGG terms in the BC vs AC comparison (Fig. 9c and d). Interestingly, "sensory system" was detected significantly enriched only in the $\mathrm{BC}$ vs AC comparison.

Based on the mRNA-miRNA interaction network in the BZ/AZ comparison of $M$. savatieri, novel_mir65 contained the largest number of co-differentially expressed target genes, of which 21 and 147 associated genes targeted by novel_mir65 were negatively and positively expressed, respectively (Figs. 10, 11 and Additional file 6). Moreover, novel_mir40 had 11 co-differentially expressed target genes, with the second largest number of correlated miRNAs in the negatively regulated interaction network. Regarding the positively regulated interaction network, novel_mir87 and novel_mir40 had 26 and 24 co-differentially expressed target genes, being the second and third largest number of correlated miRNAs, respectively.

Correlation analysis of transcriptome and miRNA data revealed several functional classifications specific to BZ/ AZ. Therefore, miRNA-target gene pairs related to these functions were screened from the described interaction networks. Finally, 10 miRNA-target gene pairs were obtained from 7 miRNAs. The novel_mir65 had 4 target genes: CL4768. Contig1 was involved in the auxinactivated signaling pathway; Unigene 36,442 was involved in the phosphorelay signal transduction system; Unigene 25,492 was associated with ATP binding; and CL5625. Contig5 was related to DNA binding. The novel_mir80 had a target gene, Unigene14700, involved in the cell surface receptor signaling pathway; novel_ mir40 had a target gene (Unigene 21,854) related to the phosphorelay signal transduction system; miR397-5p_1 had a target gene (Unigene 652) related to hydroquinone: oxygen oxidoreductase activity; novel_mir17 had a target gene (CL2077. Contig6) related to RNA polymerase II transcription cofactor activity; the target genes of novel_mir36 (CL5994. Contig7) and novel_mir25 (Unigene 13,724) were associated with MAP kinase activity (Fig. 12). These seven miRNAs may affect the parasitic development of $M$. savatieri by regulating the related genes of signaling, cellular junctions, and molecular regulation.

Table 5 Correlation results of co-differentially expressed miRNAs and target genes in M. savatieri

\begin{tabular}{|c|c|c|c|c|c|c|c|c|c|}
\hline \multirow{2}{*}{$\begin{array}{l}\text { Diff } \\
\text { group }\end{array}$} & \multicolumn{3}{|l|}{ Total } & \multicolumn{3}{|l|}{ Negative } & \multicolumn{3}{|l|}{ Positive } \\
\hline & $\begin{array}{l}\text { Co-diff } \\
\text { numbers }\end{array}$ & $\begin{array}{l}\text { miRNA } \\
\text { numbers }\end{array}$ & $\begin{array}{l}\text { Target } \\
\text { numbers }\end{array}$ & $\begin{array}{l}\text { Co-diff } \\
\text { numbers }\end{array}$ & $\begin{array}{l}\text { miRNA } \\
\text { numbers }\end{array}$ & $\begin{array}{l}\text { Target } \\
\text { numbers }\end{array}$ & $\begin{array}{l}\text { Co-diff } \\
\text { numbers }\end{array}$ & $\begin{array}{l}\text { miRNA } \\
\text { numbers }\end{array}$ & $\begin{array}{l}\text { Target } \\
\text { numbers }\end{array}$ \\
\hline$A C / B Z$ & 83 & 28 & 83 & 36 & 15 & 36 & 36 & 17 & 36 \\
\hline$A Z / A C$ & 0 & 0 & 0 & 0 & 0 & 0 & 0 & 0 & 0 \\
\hline$B C / A C$ & 78 & 30 & 77 & 21 & 11 & 21 & 36 & 21 & 35 \\
\hline $\mathrm{BC} / \mathrm{AZ}$ & 225 & 51 & 219 & 55 & 23 & 53 & 168 & 34 & 164 \\
\hline BC/BZ & 20 & 14 & 19 & 1 & 1 & 1 & 19 & 14 & 18 \\
\hline $\mathrm{BZ} / \mathrm{AZ}$ & 320 & 53 & 310 & 58 & 22 & 57 & 260 & 39 & 251 \\
\hline
\end{tabular}

BC, growing without a host for 8 WAS; BZ, growing with one G. jasminoides for 8 WAS; AC, growing without a host for 16 WAS; AZ, growing with one G. jasminoides for 16 WAS 


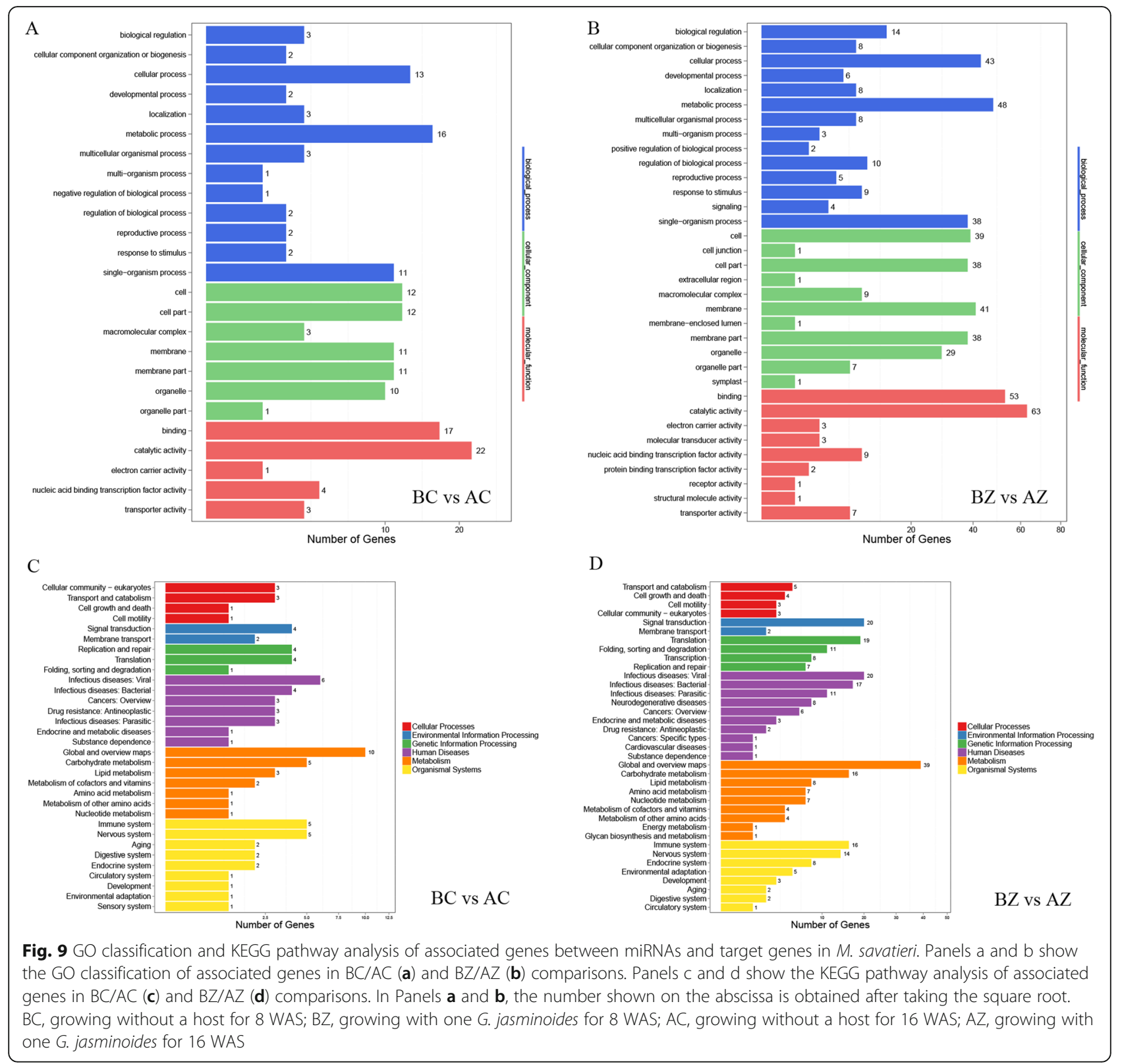

\section{Discussion}

M. savatieri is a medically important parasite, and much attention has been given to artificial cultivation because of its sharply declining wild resources. The essence of life habits in parasites is to establish parasitic relationships with the host [23]. In recent decades, research on the establishment of parasitic relationship has revealed its role in promoting the growth and development of some hemiparasitic plants including Pedicularis rex and Thesium chinense $[24,25]$. In our recent study, the growth and development of $M$. savatieri was significantly promoted after parasitizing the host (Fig. 1a). However, to date, there has been no study on the underlying molecular mechanisms, and little is known about the characteristics of related endogenous hormones. This study is the first to explore the changes in the transcriptome and small RNA before and after establishing parasitic relationships with the host.

Phytohormones are important factors regulating plant growth and development. By analyzing the KEGG pathway, many DEGs were found to be enriched in "plant hormone signal transduction" (Fig. $3 \mathrm{e}$ and $\mathrm{f}$ ). The present study showed that levels of auxins such as IAA, ME-IAA, and ICA showed a significant decrease with $M$. savatieri development, independent of establishing the parasite-host association (Fig. 1b and c). Similarly, a 

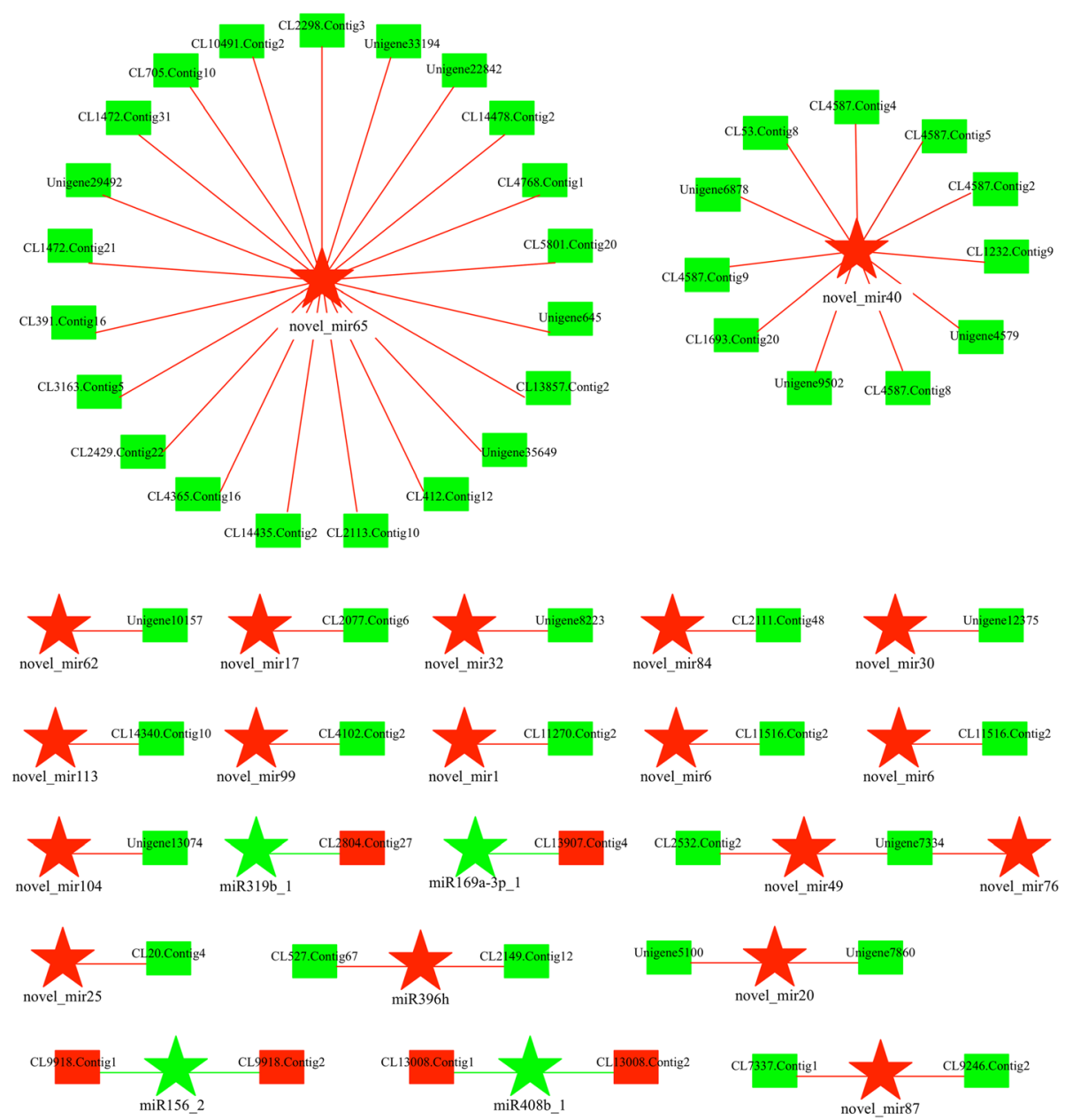

Fig. 10 The interaction network of co-differentially negatively expressed miRNAs combined with mRNAs in the BZ/AZ comparison of M. savatieri. The stars represent miRNAs and rectangle nodes represent mRNAs. Red represents the up-regulated nodes, and green represents the downregulated nodes. BZ, growing with one G. jasminoides for 8 WAS; AZ, growing with one G. jasminoides for 16 WAS

downtrend of IAA content was detected in Cuscuta japonica during its parasitization [11]. Although JA and SA levels in $M$. savatieri grown without the host increased significantly with development, contrary variation was observed in seedlings grown with the host (Fig. 1e and f). JA is reported to regulate plant growth and stress responses [26], and SA is found to mediate stress tolerance [27]. However, the functions of JAs and SA in parasitic plants are still unknown. We speculate that JAs and SA may play a crucial role in stressacclimation signaling, and the decreased JA and SA levels in attached M. savatieri seedlings may be related to a reduced demand for protection. Increasing ABA levels in attached hemiparasites have been reported in Rhinanthus minor [28]. ABA levels in M. savatieri followed a similar trend, increasing significantly after parasitizing the host compared with seedlings grown with the host for 8 WAS (Fig. 1f). The high levels of ABA in attached hemiparasites may be due to the increase in selfsynthesized ABA and ABA obtained from the host [29]. Surprisingly, M. savatieri grown for 16 WAS had approximately 3.7 times more ABA than $M$. savatieri grown for 8 WAS even when growing without the host. It is possible that parasites that have been unattached for a long time were susceptible to abiotic stress, thus stimulating the increase in ABA, which is a plant drought-related hormone. These results provide a clue for improving the growth of beneficial parasites by exogenous application of plant growth regulators, although further evidence needs to be obtained to verify the effects.

In this study, most DEGs associated with "starch and sucrose metabolism" were enriched in the comparisons of M. savatieri plants with established parasitic relationships (Fig. 3e and f). Moreover, associated genes between miRNAs and target genes were also involved in "carbohydrate metabolism" of M. savatieri (Fig. 9c and d). 




Fig. 11 The interaction network of co-differentially positively expressed miRNAs combined with mRNAs in the BZ/AZ comparison of M. savatieri. The stars represent miRNAs and rectangle nodes represent mRNAs. Red represents the up-regulated nodes, and green represents the downregulated nodes. BZ, growing with one G. jasminoides for 8 WAS; AZ, growing with one G. jasminoides for 16 WAS

Similarly, unigenes associated with carbohydrate, starch and sucrose metabolism have been identified in the development of the parasite Arceuthobium sichuanense at the transcriptional level [30]. Combined with the results of our research, these data collectively suggest that the metabolism of carbohydrates, starch and sucrose could have an essential role in the development of $M$. savatieri after establishing parasitic relationships with the host. In our recent research, the observation that the growth of $M$. savatieri in the absence of a host plant was increased under the condition of sufficient nitrogen was first reported [31]. Interestingly, the present study showed that the DEGs significantly enriched in "nitrogen metabolism" and "photosynthesis" were observed only in the development of $M$. savatieri grown without the host (Fig. 3a). Considering that the increasing nitrogen supply stimulated the photosynthetic rates of Striga hermonthica [32], and the retained photosynthetic capacity in $M$. savatieri, we speculated that nitrogen metabolism may be closely related to photosynthesis during autotrophic growth, which might suggest a survival strategy for root hemiparasites in the absence of the host. Furthermore, the DEGs and DEmiR targets involved in "carbon fixation in photosynthetic organisms" were enriched only in the development of $M$. savatieri, which growing without G. jasminoides (Figs. 3a, 8a). The findings that most carbon used by the parasites came from the host may partially explain these results [33], suggesting that $M$. savatieri largely depended on the photosynthesis of carbon before attaching to the host.

Based on transcriptome and small RNA sequencing in $M$. savatieri, the correlation analysis demonstrated that most co-differentially expressed miRNAs and targets were represented in the comparisons of plants with 


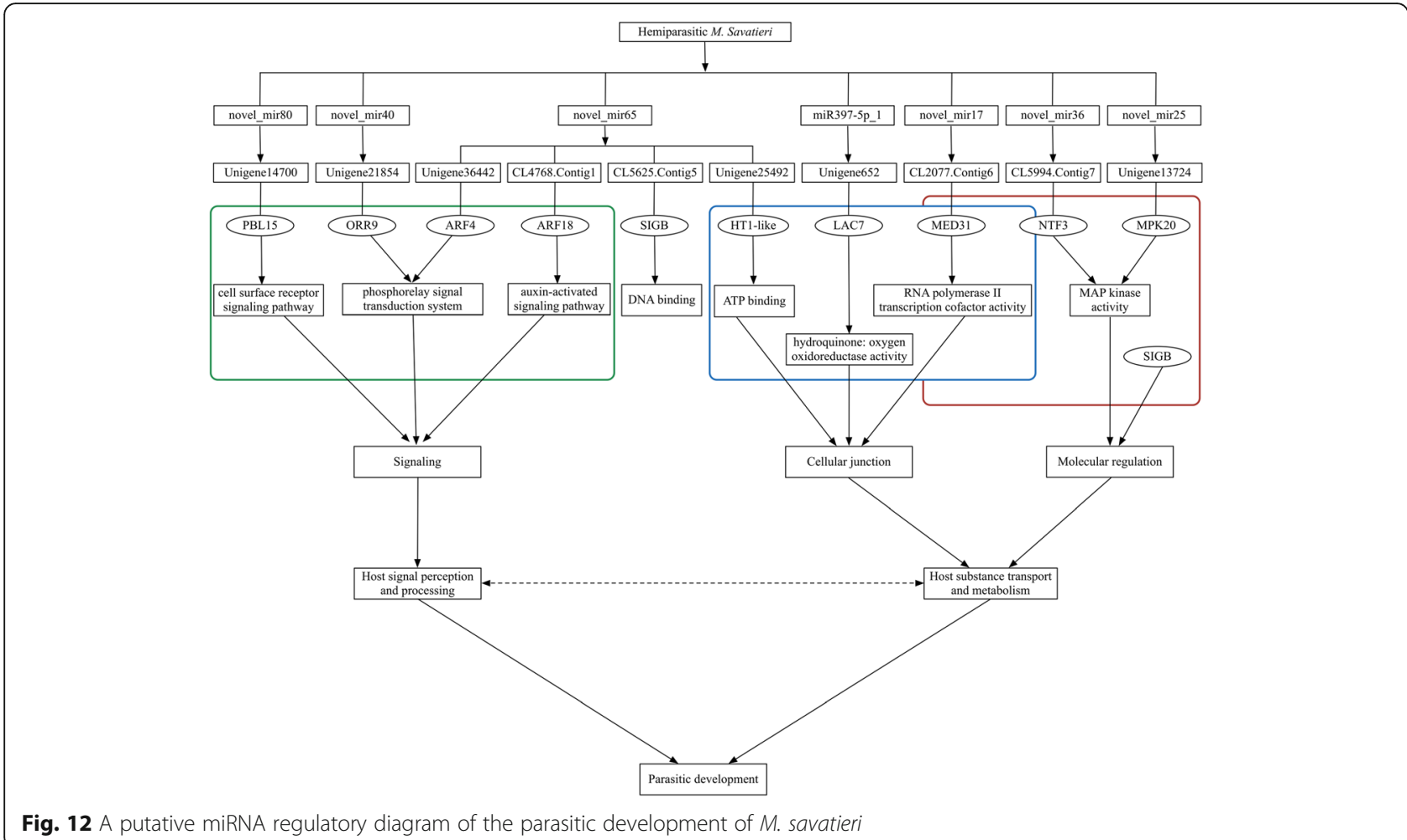

Fig. 12 A putative miRNA regulatory diagram of the parasitic development of M. savatier

established parasitic relationships (Table 5). In previous studies, mRNAs were found to move bidirectionally between parasites and hosts [16], and parasite miRNAtargeted host mRNAs act as trans-species regulators during parasitism [22], implying that genetic molecule exchange may also take place in $M$. savatieri-host plant interactions, and most miRNAs and targets play significant roles in the post-parasitization stage. Notably, neither positive nor negative correlations were found in the co-differentially expressed miRNAs and targets in the AC vs AZ comparison (Table 5). The results are not in line with our expectations. Further transcriptome and small RNA sequencing of the host plant will be useful to understand the mechanisms underlying the results and verify $M$. savatieri-host plant interactions. In addition, the associated targets participating in "cell junction", "extracellular region", "membrane-enclosed lumen" and "symplast" were differentially expressed in $M$. savatieri plants before and after establishing parasitic relationships with G. jasminoides (Fig. 9a and b). The symplastic or apoplastic transport of nutrient acquisition from the host plant by the parasite has been discussed in recent years [34]. The analysis of RNA-seq and sRNA-seq demonstrated that the transport mechanism of $M$. savatieriacquired nutrients from the host plant included symplast in addition to apoplast. The result was similar to the finding reported for C. pentagona [14].
There were 162 differentially expressed known and novel miRNAs identified in $M$. savatieri before and after establishing parasitic relationships with the host (Fig. 7a); however, only 53 DEmiRs led their target genes to exhibit significantly different expression (Figs. 10 and 11). Considering the role of phased small interfering RNA (siRNA) in growth regulation [35], we speculated that siRNA may also act as a regulator in the gene expression of $M$. savatieri development. The roles of miRNAs in regulating plant development have been studied extensively. In Arabidopsis, miR167 regulates the expression pattern of the ARF6 and ARF8 genes and is essential for the fertility of ovules and anthers [36]. Overexpression of miR396 results in a reduction of growth-regulating factor expression and the cell number in leaves of $A$. thaliana [37]. In this mRNA-miRNA interaction network, the known miRNAs belonging to miR167, miR396 and miR845 families, and their targets were co-differentially expressed in the roots of $M$. savatieri before and after establishing parasitic relationships (Figs. 10 and 11). Of these miRNAs, most known miRNAs were conserved among plant species. However, miR845 has not been commonly reported in plants, suggesting that some miRNAs may be specific to some species. Moreover, miR396 was also one of the most numerous families in M. savatieri (Fig. 6c), which suggested that miR396 might have a fundamental role in 
the processes of parasite development. However, based on the mRNA-miRNA interaction network, most codifferentially expressed genes were targeted by some novel miRNAs, including novel_mir65, novel_mir40 and novel_mir87 (Figs. 10 and 11). Therefore, further molecular experiments should be conducted to explore their roles and mechanisms during parasitism of M. savatieri.

\section{Conclusions}

Establishing parasitic relationships with the host decreased the levels of IAA and JA and increased the ABA content in $M$. savatieri. Our study provided the first transcriptome and small RNA resources for further investigations of the responsible molecular mechanisms of enhanced growth in M. savatieri after establishing parasitic relationship. Using high-throughput sequencing, transcriptome data consisting of 167,941 unigenes were obtained from $M$. savatieri roots, and 46,424 DEGs were detected in the comparison between $M$. savatieri with the established parasitic relationship and without the established parasitic relationship. Analysis of small RNA data obtained 128 novel miRNAs and 110 known miRNAs, and 198 miRNAs were significantly differentially expressed in $M$. savatieri. The DEGs and DEmiRs related to growth and development in $M$. savatieri were revealed by the GO and KEGG pathway enrichment analysis. Moreover, correlation analysis of mRNA and miRNA revealed that 10 miRNA-target pairs from novel_mir65, novel_mir40, novel_mir80, miR397-5p_1, novel_mir36, novel_mir25 and novel_mir17 may have vital roles in regulating the parasitic development of $M$. savatieri. The data generated by this study provided abundant information and gene and miRNA sequences to accelerate the molecular and genetic studies in $M$. savatieri. In the future, research is needed to know the exact mechanisms by which the establishment of parasite-host associations regulates growth and physiological processes.

\section{Methods}

\section{Plant materials and growth conditions}

M. savatieri seed capsules at fully mature were collected from the experimental base, located at Yongfeng County, Jiangxi Province, of the Huizhou Jiuhui Pharmaceutical Co., Ltd., in May 2017 (the collection was permitted by Huizhou Jiuhui Pharmaceutical Co., Ltd.), and then stored in paper bags at $4{ }^{\circ} \mathrm{C}$ until required. M. savatieri was identified and authenticated by Professor Qiaosheng Guo, Nanjing Agricultural University. The voucher specimen was deposited in the Institute of Chinese Medicinal Materials, Nanjing Agricultural University (Nanjing, China). Due to Gardenia jasminoides E.
(Rubiaceae) was widely distributed in the plant community of $M$. savatieri [3] and could be parasitized by potted $M$. savatieri [7], it was used as a host in this experiment. $M$. savatieri seedlings were grown in the presence or absence of one G. jasminoides plant. On March 19, 2018, after washing with tap water, we transplanted the commercially available G. jasminoides plants of about $15 \mathrm{~cm}$ in height (purchased from Lingshan Yufeng Guomiao Co., Ltd) into the pot filled with $770 \mathrm{~g}$ of fine sand and nutritive soil $(2: 1, \mathrm{v} / \mathrm{v})$, one G. jasminoides plant per pot. In order to promote seed germination, the $800 \mathrm{mg} \cdot \mathrm{L}^{-1}$ gibberellin solution was used to soak M. savatieri seeds for $24 \mathrm{~h}$ at room temperature [38]. These seeds were sown on April 17, 2018, and each pot contained 50 seeds. Thinning was carried out at 4 weeks after sowing (WAS), and 10 seedlings of about $5 \mathrm{~mm}$ in height were kept in each pot [7].

All plants were cultivated in a natural night/day greenhouse at the Institute of Chinese Medicinal Materials, Nanjing Agricultural University. This experiment was conducted for 20 weeks from spring (March, 2018) to summer (August, 2018). After thinning (from May to August), the greenhouse was covered with a 50\% SunBlock shade net to make the light condition similar to that of M. savatieri habitats. During the whole experiment, the pots were watered with tap water every day to reach field capacity and placed fully at random, and were rerandomized every 2 weeks to minimize the effect of position.

\section{Harvest and sampling}

Two harvests were conducted to examine the differences between $M$. savatieri grown in the presence or absence of G. jasminoides at two key developmental phases of the parasitic plants [23]. One at 8 WAS (early seedling phase, before establishing parasitic relationship with the host), and the other at 16 WAS (late seedling phase, after establishing parasitic relationship with the host). The obvious enhancement of hemiparasite growth confirmed the successful establishment of $M$. savatieri-G. jasminoides association [39]. The experiment included four different groups. BC, M. savatieri that had grown without G. jasminoides for 8 WAS; BZ, M. savatieri that had grown with G. jasminoides for 8 WAS; AC, M. savatieri that had grown without G. jasminoides for 16 WAS; AZ, M. savatieri that had grown with G. jasminoides for 16 WAS. For analysis of plant endogenous hormones, seedling samples from each group were collected with three replicates and weighed for further measurement. For analysis of transcriptome and small RNA, M. savatieri roots collected from each group were quickly frozen in liquid nitrogen and then stored at $-80^{\circ} \mathrm{C}$ until required. There were three replicates for transcriptome and small RNA analysis. 


\section{Endogenous hormone measurement}

The extraction, purification and measurement of IAA, ME-IAA, IC), tZ, cZ, JA, H2JA, jasmonoyl-(L)-isoleucine (JA-ILE), SA and ABA were performed as previously reported [40]. Briefly, $1 \mathrm{~g}$ of fresh weight of $M$. savatieri samples was extracted with $10 \mathrm{~mL}$ methanol/water/formic acid $(15: 4: 1, \mathrm{v} / \mathrm{v} / \mathrm{v})$ at $4{ }^{\circ} \mathrm{C}$. The LC-ESI-MS/MS system (MS, Applied Biosystems 6500 Triple Quadrupole; HPLC, Shim-pack UFLC SHIMADZU CBM30A system) was utilized to analyze the generated extracts, with the ESI-triple quadrupole-linear ion trap (Q TRAP)-MS alternatively connected to the effluent. In addition, the API 6500 Q TRAP LC/MS/MS System controlled by Analyst 1.6 software (AB Sciex) was equipped with the ESI Turbo Ion-Spray interface and operated in positive ion mode.

Total RNA extraction, library construction and sequencing Total RNA extraction of the root tissues in M. savatieri was performed using the TRIzol reagent kit (Invitrogen, USA) based on the manufacturer's guidance. The Nanodrop 8000 spectrophotometer (Thermo Fisher Scientific, USA) and Agilent 2100 Bioanalyzer (Agilent Technologies, USA) were used to evaluate the quantity and quality of these total RNAs, respectively. The qualified total RNA was ready for transcriptome and small RNA sequencing using the BGISEQ-500 platform. A total of 12 mRNA and sRNA libraries were constructed with three biological replicates for each treatment. Sequencing, data processing, gene functional annotation, and miRNA identification were conducted by BGI (Shenzhen, China). Both RNA-seq and small RNA-seq data have been deposited in the NCBI SRA database, with BioProject IDs PRJNA600790 and PRJNA601043, respectively. Trinity software (parameter: --min_contig_length 150 --CPU 8 --min_kmer_cov 3 --min_glue 3 --bfly_opts '-V 5 --edge-thr $=0.1$--stderr') was adopted to de novo assembly of the filtered clean reads [41]. In addition, we performed BUSCO with default parameters to check the quality of the assembled transcripts [42].

\section{Transcriptome and small RNA analysis}

The known miRNAs were identified by matching the sequences to the known mature miRNAs in the miRBase. Based on the characteristic hairpin secondary structure of the miRNA precursor, miRA [43] was adopted to predict novel miRNA using default parameters (cluster min_reads $=10$; min_precursor_length $=50 ;$ max $\_$mfe per_nt $=-0.2 ; \quad$ min_duplex_length $=18 ; \quad$ max_duplex length $=30$; allow_three_mismatches $=1$ ). The DESeq $R$ package (1.18.0) was used for analysis of the differential expression of pairs. To control the false discovery rate, the approach of Benjamini and Hochberg was adopted to adjust the $P$-value. The conditions used to screen the
DEGs and DEmiRs were a fold change $\geq 2.00$ and an adjusted $P$-value $<0.05$ [44]. GO and KEGG enrichment analyses of the DEGs and target genes of DEmiRs were implemented based on the hypergeometric model [45]. To obtain more accurate results, both psRobot (parameter: -gl 17 -p 8 -gn 1) [46] and TargetFinder (parameter: -c 4) [47] were adopted to predict the target genes of miRNAs, and the corresponding intersection was selected as the final result.

\section{Analysis of DEG network}

To perform the analysis of network relations, the DEGs were compared with the STRING database by DIAMOND, and the interaction between genes was obtained by homology with known proteins. There is a score value for the interaction between genes, which indicates the reliability of the interaction. From 150 to 1000, the higher the score, the more reliable the interaction relationship. In our analysis, the network relations with score $\geq 300$ were selected for building the DEG network.

\section{Correlation analysis of mRNA and miRNA}

To further understand all the possible interactions between mRNA and miRNA (negative and positive correlations of mRNA-miRNA expression), an mRNA-miRNA regulatory network was built using the in-house $\mathrm{R}$ script. In brief, to integrate the DEmiRs with DEGs, matched mRNA and miRNA sequencing data for all samples were normalized, and then the expression profiles of mRNA and miRNA, the information for the miRNA target, and sample categories were integrated.

\section{Quantitative real-time PCR analysis}

In the experiment, 14 genes, 15 miRNAs and target genes associated with "cell part", "plant hormone signal transduction", and "metabolism" were selected to analyze the expression levels by qRT-PCR. Based on the instructions of the manufacturer, the TaKaRa MiniBEST Plant RNA Extraction Kit (TaKaRa, China) was adopted to extract the total RNA from BC, BZ, AC and AZ. The gDNA Eraser Perfect Real Time and PrimeScript ${ }^{\mathrm{im}} \mathrm{RT}$ reagent Kit (TaKaRa, China) were adopted to synthesize cDNA used for the qRT-PCR analysis of genes and miRNA targets. The miRNA expression was validated by RNA-tailing, and cDNA used for the qRT-PCR analysis of miRNAs was synthesized using the Mir- $\mathrm{X}^{\mathrm{m}}$ miRNA First-Strand Synthesis and TB Green ${ }^{\text {Tx }}$ qRT-PCR Kit (TaKaRa, China). The amplified reaction was conducted with the Step One Real-Time system (Applied Biosystems, USA) based on a previous report [48]. Three biological replicates were applied. All primer sequences are given in Tables S2, S3 and S4. For the genes and miRNA targets, the relative expression levels were normalized against the geometric average of the GAPDH and actin 
genes [49]. For the miRNAs, U6 snRNA served as the internal control, and the analysis was carried out based on the $2^{-\Delta \Delta \mathrm{CT}}$ method [50].

\section{Statistical analysis}

IBM Statistical Product and Service Solutions (SPSS) 22.0 software (IBM Corporation, USA) was used to perform the statistical analysis, and the means were compared by Duncan's multiple range test $(P<0.05)$.

\section{Supplementary Information}

The online version contains supplementary material available at https://doi. org/10.1186/s12870-021-02861-6.

Additional file 1: Fig. S1. Unigene sequence length distribution in the transcriptome of $M$. savatieri. Fig. S2. GO classification of all unigenes in M. savatieri. Fig. S3. Heatmap clustering and qRT-PCR analysis of DEGs in M. savatieri. (a) Transcript abundances of DEGs related to starch and sucrose metabolism. (b) Relative expression levels of DEGs related to starch and sucrose metabolism. (c) Transcript abundances of DEGs related to hormone signal transduction. (d) Relative expression levels of DEGs related to hormone signal transduction. (e) Transcript abundances of DEGs related to cell part. (f) Relative expression levels of DEGs related to cell part. The error bars of qRT-PCR data indicate the standard deviations of the three replicate determinations. Fig. S4. Nucleotide length distribution of small RNAs in M. savatieri. Fig. S5. The first base distribution of known miRNAs (a) and novel miRNAs (b) in M. savatieri. In the bar graphs, the $\mathrm{X}$-axis represents the length of miRNA, the number shown on the bar represents the number of miRNA at this length. Fig. S6. Heatmap clustering and GRT-PCR analysis of miRNAs in M. savatieri. (a) Sequencing abundances of miRNAs related to biological regulation. (b) Relative expression levels of miRNAs related to biological regulation. (c) Sequencing abundances of miRNAs related to membrane and organelle. (d) Relative expression levels of miRNAs related to membrane and organelle. (e) Sequencing abundances of miRNAs related to metabolism. (f) Relative expression levels of miRNAs related to metabolism. The error bars of qRTPCR data indicate the standard deviations of the three replicate determinations. Fig. S7. qRT-PCR analysis of miRNA target genes in M. savatieri. The error bars of qRT-PCR data indicate the standard deviations of the three replicate determinations. Table S1. Transcription factor families of the DEGs related to the biological processes in the BZ/AZ comparison of M. savatieri. Table S2. Specific primers of differentially expressed genes for qRT-PCR validation. Table S3. Specific primers of differentially expressed miRNAs for qRT-PCR validation. Table S4. Specific primers of target genes of differentially expressed miRNAs for qRT-PCR validation.

Additional file 2. List of DEGs among different comparisons. Additional file 3. GO classification of DEGs among different comparisons.

Additional file 4. List of DEmiRs among different comparisons.

Additional file 5. KEGG pathway of DEmiR target genes among different comparisons.

Additional file 6. Co-differentially expressed miRNAs and targets in the $B C / A C$ and BZ/AZ comparisons.

\section{Abbreviations}

RNA-seq: RNA sequencing; DEGs: Differentially expressed genes; DEmiRs: Differentially expressed miRNAs; NR: Non-redundant protein database; NT: Nucleotide sequence database; GO: Gene Ontology; KEGG: Kyoto Encyclopedia of Genes and Genomes; sRNAs: Small RNAs; WAS: Weeks after sowing; IAA: Indole-3-acetic acid; ME-IAA: Methyl indole-3acetate; ICA: Indole-3-carboxaldehyde; tZ: Trans-zeatin; cZ: Cis-zeatin; JA: Jasmonic acid; H2JA: Dihydrojasmonic acid; JA-ILE: Jasmonoyl-(L)isoleucine; SA: Salicylic acid; ABA: Abscisic acid; qRT-PCR: Quantitative realtime $P C R$

\section{Acknowledgements}

We thank Mr. Kai Long from Aviation University Air Force for his assistance with the image processing during the preparation of the manuscript.

\section{Authors' contributions}

LC, ZZ, and QG designed the experiments; LC performed the experiments and wrote the original draft; $Z Z$ and $Q G$ revised the manuscript; $H W$ and YQ contributed cultivation experimental materials; LC and $\mathrm{HZ}$ analyzed the data. All authors read and approved the manuscript.

\section{Funding}

The research was financially supported by the Huizhou Jiuhui Pharmaceutical Co., Ltd. (Grant No. HY0068). The funding body had no role in the design of the study, collection, analysis, and interpretation of data, and in writing the manuscript. Seed capsules of M. savatieri were collected from an experimental base of the Huizhou Jiuhui Pharmaceutical Co., Ltd.

\section{Availability of data and materials}

The RNA-seq read data analyzed during the current study are available in the NCBI SRA database, https://www.ncbi.nlm.nih.gov/sra/PRJNA600790 (accession BioProject number: PRJNA600790). The Small RNA-seq read data analyzed during the current study are available in the NCBI SRA database, https://www.ncbi.nlm.nih.gov/sra/PRJNA601043 (accession BioProject number: PRJNA601043).

\section{Ethics approval and consent to participate}

Not applicable.

\section{Consent for publication}

Not applicable.

\section{Competing interests}

The authors declare that they have no competing interests.

Received: 23 June 2020 Accepted: 28 January 2021

Published online: 10 February 2021

\section{References}

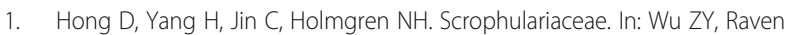
$\mathrm{PH}$, editors. Flora of China. Beijing: Science Press and Missouri Botanical Garden Press; 1998. p. 211-2.

2. Yamazaki T. Scorphulariaceae. In: Iwatsuki K, Yamazaki T, Bufford DE, Ohba H, editors. Flora of Japan. Tokyo: Smithsonian Institution Press; 1993. p. 358-9.

3. Zhang MH, Chen YL, Ouyang Y, Huang ZG, Silva JAT, Ma GH. The biology and haustorial anatomy of semi-parasitic Monochasma savatieri Franch. Ex maxim. Plant Growth Regul. 2015;75:473-81.

4. Committee of National Pharmacopoeia, Pharmacopoeia of P.R. China. Pharmacopoeia of the People's Republic of China. Beijing: China Medical Science Press; 2015. p. 1150-1.

5. Yang YZ. Plantlet regeneration of Monochasma savatieri Franch. Ex maxim In vitro culture from inducing adventitious root. Plant Physiol Commun. 2009;7:693-4.

6. Zhang YY, Chen YL, Zhang XH, Silva JAT, Ma GH. Adventitious shoot induction from internode and root explants in a semiparasitic herb Monochasma savatieri Franch ex maxim. J Plant Growth Regul. 2017;36: 799-804.

7. Chen LL, Zhu ZB, Guo QS, Guo J, Huang ZG, Zhang H. The changes of morphological and physiological characteristics in hemiparasitic Monochasma savatieri before and after attachment to the host plant. PeerJ. 2020;8:e9780

8. Kuijt J. The biology of parasitic flowering plants. Berkeley: The University of California Press; 1969.

9. Yao DR, Zheng XM, Huang JZ, Li YH. Changes of acid phosphatase and cytokinins during haustorial development of the parasitic plant Cassytha Filiformis L. Acta Bot Sin. 1994:36:170-4.

10. Huang JZ, Li YH. Relationship between haustorial formation of Cuscuta japonica induced by exogenous cytokinin and calmodulin. Acta Bot. BorealOccident Sin. 1991;11:116-20. 
11. Löffler C, Czygan FC, Proksch P. Role of indole-3-acetic acid in the interaction of the phanerogamic parasite Cuscuta and host plants. Plant Biol. 1999;1:613-7.

12. Honaas LA, Wafula EK, Yang ZZ, Der JP, Wickett NJ, Altman NS, Taylor CG, Yoder Jl, Timko MP, Westwood JH, de Pamphilis CW. Functional genomics of a generalist parasitic plant: laser microdissection of host-parasite interface reveals host-specific patterns of parasite gene expression. BMC Plant Biol. 2013;13:9.

13. Wickett NJ, Honaas LA, Wafula EK, Das M, Huang K, Wu B, Landherr L, Timko MP, Yoder J, Westwood JH, de Pamphilis CW. Transcriptomes of the parasitic plant family Orobanchaceae reveal surprising conservation of chlorophyll synthesis. Curr Biol. 2011;21:2098-104.

14. Ranjan A, Ichihashi Y, Farhi M, Zumstein K, Townsley B, David-Schwartz R, Sinha NR. De novo assembly and characterization of the transcriptome of the parasitic weed Cuscuta pentagona identifies genes associated with plant parasitism. Plant Physiol. 2014;166:1186-99.

15. Zhang X, Berkowitz O, Silva JAT, Zhang M, Ma G, Whelan J, Duan J. RNA-Seq analysis identifies key genes associated with haustorial development in the root hemiparasite Santalum album. Front Plant Sci. 2015;6:661.

16. Kim G, LeBlanc ML, Wafula EK, de Pamphilis CW, Westwood JH. Genomicscale exchange of mRNA between a parasitic plant and its hosts. Science. 2014;345:808-11.

17. Fabian MR, Sonenberg N, Filipowicz W. Regulation of mRNA translation and stability by microRNAs. Annu Rev Biochem. 2010;79:351-79.

18. Obernosterer G, Leuschner PJ, Alenius M, Martinez J. Post-transcriptional regulation of microRNA expression. RNA. 2006;12:1161-7.

19. Chen XM. A microRNA as a translational repressor of APETALA2 in Arabidopsis flower development. Science. 2004;303:2022-5.

20. Wang JW, Park MY, Wang LJ, Koo Y, Chen XY, Weigel D, Poethig RS. miRNA control of vegetative phase change in trees. PLoS Genet. 2011;7:e1002012.

21. Zhang L, Hou D, Chen X, Li D, Zhu L, Zhang Y, Li J, Bian Z, Liang X, Cai X, Yin Y, Wang C, Zhang T, Zhu D, Zhang D, Xu J, Chen Q, Ba Y, Liu J, Wang Q, Chen J, Wang J, Wang M, Zhang Q, Zhang J, Zen K, Zhang C. Exogenous plant MIR168a specifically targets mammalian LDLRAP1: evidence of crosskingdom regulation by microRNA. Cell Res. 2012;22:107-26.

22. Shahid S, Kim G, Johnson NR, Wafula E, Wang F, Coruh C, Bernal-Galeano V, Phifer T, de Pamphilis CW, Westwood JH, Axtell MJ. MicroRNAs from the parasitic plant Cuscuta campestris target host messenger RNAs. Nature. 2018;553:82-5

23. Joel DM. The haustorium and the life cycles of parasitic Orobanchaceae. In: Joel DM, Gressel J, Musselman J, editors. Parasitic Orobanchaceae. Heidelberg: Springer; 2013. p. 22.

24. Guo QS, Luo FL. Comparative studies on the growth, chlorophyll, amino acids and minerals of Thesium chinense (Santalaceae) in association with different hosts. Nord J Bot. 2010:28:632-40.

25. Li AR, Smith FA, Smith SE, Guan KY. Two sympatric root hemiparasitic Pedicularis species differ in host dependency and selectivity under phosphorus limitation. Funct Plant Biol. 2012;39:784-94.

26. León J, Sánchez-Serrano JJ. Molecular biology of jasmonic acid biosynthesis in plants. Plant Physiol Biochem. 1999;37:373-80.

27. Pan Q, Zhan J, Liu H, Zhang J, Chen J, Wen P, Huang W. Salicylic acid synthesized by benzoic acid 2-hydroxylase participates in the development of thermotolerance in pea plants. Plant Sci. 2006;171:226-33.

28. Jiang F, Jeschke WD, Hartung W. Water flows in the parasitic association Rhinanthus minor/Hordeum vulgare. J Exp Bot. 2003:54:1985-93.

29. Westwood JH. The physiology of the established parasite-host association. In: Joel DM, Gressel J, Musselman J, editors. Parasitic Orobanchaceae. Heidelberg: Springer; 2013. p. 87-114.

30. Wang Y, Li X, Zhou W, Li T, Tian C. De novo assembly and transcriptome characterization of spruce dwarf mistletoe Arceuthobium sichuanense uncovers gene expression profiling associated with plant development. BMC Genomics. 2016;17:771.

31. Chen L, Zhu Z, Guo Q, Tang S, Huang Z, Zhong W, Wen Z. Growth response of Monochasma savatieri to nitrogen, phosphorus, and potassium in the absence of a host plant: essential demand for nutrients of root hemiparasitic plants. J Plant Nutr. 2020;43:444-55.

32. Cechin I, Press MC. Nitrogen relations of the sorghum-Striga hermonthica host-parasite association: growth and photosynthesis. Plant Cell Environ. 1993;16:237-47.
33. Irving LJ, Kim D, Schwier N, Vaughan JKE, Ong G, Hama T. Host nutrient supply affects the interaction between the hemiparasite Phtheirospermum japonicum and its host Medicago sativa. Environ Exp Bot. 2019;162:125-32.

34. Birschwilks M, Haupt S, Hofius D, Neumann S. Transfer of phloem-mobile substances from the host plants to the holoparasite Cuscuta sp. J Exp Bot. 2006:57:911-21.

35. Chen C, Zeng Z, Liu Z, Xia R. Small RNAs, emerging regulators critical for the development of horticultural traits. Hortic Res. 2018:5:63.

36. Wu MF, Tian Q, Reed JW. Arabidopsis microRNA167 controls patterns of ARF6 and ARF8 expression, and regulates both female and male reproduction. Development. 2006;133:4211-421.

37. Rodriguez RE, Mecchia MA, Debernardi JM, Schommer C, Weigel D, Palatnik JF. Control of cell proliferation in Arabidopsis thaliana by microRNA miR396. Development. 2010;137:103-12.

38. Chen YF, Guo QS, Zhu ZB, Chen LL, Tang SQ, Huang ZG, Wen ZC. Effects of gibberellin concentration and temperature on seeds germination of Monochasma savatieri. J Chinese Med Mater. 2018:41:1040-3.

39. Sui XL, Zhang T, Tian YQ, Xue RJ, Li AR. A neglected alliance in battles against parasitic plants: arbuscular mycorrhizal and rhizobial symbioses alleviate damage to a legume host by root hemiparasitic Pedicularis species. New Phytol. 2019;221:470-81.

40. Hao YH, Zhang Z, Wang L, Liu C, Lei AW, Yuan BF, Feng YQ. Stable isotope labeling assisted liquid chromatography-electrospray tandem mass spectrometry for quantitative analysis of endogenous gibberellins. Talanta. 2015;144:341-8.

41. Grabherr MG, Haas BJ, Yassour M, Levin JZ, Thompson DA, Amit I, Adiconis X, Fan L, Raychowdhury R, Zeng Q, Chen Z, Mauceli E, Hacohen N, Gnirke A, Rhind N, Palma FD, Birren B, Nusbaum C, Lindblad-Toh K, Friedma N, Regev A. Full-length transcriptome assembly from RNA-Seq data without a reference genome. Nature Biotechnolony. 2011;29:644-52.

42. Seppey M, Manni M, Zdobnov EM. BUSCO: assessing genome assembly and annotation completeness. Methods Mol Biol. 1962;2019:227-45.

43. Evers M, Huttner M, Dueck A, Meister G, Engelmann JC. miRA: Adaptable novel miRNA identification in plants using small RNA sequencing data. BMC Bioinformatics. 2015;16:370.

44. Wang L, Feng Z, Wang X, Wang X, Zhang X. DEGseq: an R package for identifying differentially expressed genes from RNA-seq data. Bioinformatics. 2010;26:136-8.

45. Boyle El, Weng S, Gollub J, Jin H, Botstein D, Cherry JM, Sherlock G. GO: TermFinder-open source software for accessing gene ontology information and finding significantly enriched gene ontology terms associated with a list of genes. Bioinformatics. 2004;20:3710-5.

46. Wu HJ, Ma YK, Chen T, Wang M, Wang XJ. PsRobot: a web-based plant small RNA meta-analysis toolbox. Nucleic Acids Res. 2012;40:W22-8.

47. Fahlgren N, Carrington JC. miRNA target prediction in plants. Method Mol. Biol. 2010;592:51-7.

48. Wang T, Zou Q, Guo Q, Yang F, Lu L, Zhang W. Widely targeted metabolomics analysis reveals the effect of flooding stress on the synthesis of flavonoids in Chrysanthemum morifolium. Molecules. 2019;24:3695.

49. Vandesompele J, Preter KD, Pattyn F, Poppe B, Roy NV, Paepe AD, Speleman F. Accurate normalization of real-time quantitative RT-PCR data by geometric averaging of multiple internal control genes. Genome Biol. 2002; 3:research0034.1-research0034.11.

50. Pfaffl MW. A new mathematical model for relative quantification in real-time RT-PCR. Nucleic Acids Res. 2001;29:e45.

\section{Publisher's Note}

Springer Nature remains neutral with regard to jurisdictional claims in published maps and institutional affiliations. 